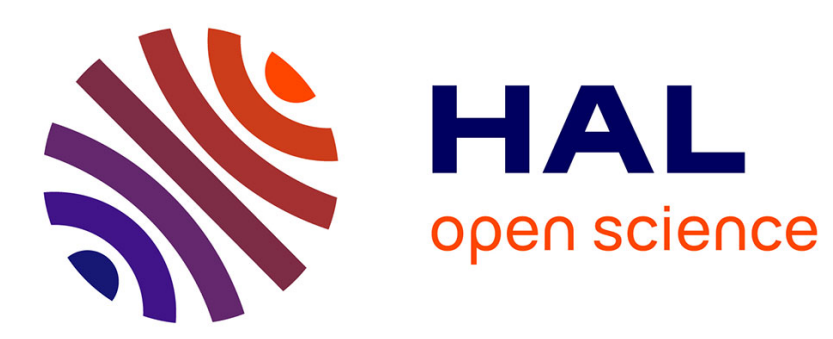

\title{
Strain-Induced Crystallization in Natural Rubber: Flory's Theory Revisited
}

Paul Sotta, Pierre-Antoine Albouy

\section{To cite this version:}

Paul Sotta, Pierre-Antoine Albouy. Strain-Induced Crystallization in Natural Rubber: Flory's Theory Revisited. Macromolecules, 2020, 53 (8), pp.3097-3109. 10.1021/acs.macromol.0c00515 . hal03016563

\section{HAL Id: hal-03016563 https://hal.science/hal-03016563}

Submitted on 20 Nov 2020

HAL is a multi-disciplinary open access archive for the deposit and dissemination of scientific research documents, whether they are published or not. The documents may come from teaching and research institutions in France or abroad, or from public or private research centers.
L'archive ouverte pluridisciplinaire HAL, est destinée au dépôt et à la diffusion de documents scientifiques de niveau recherche, publiés ou non, émanant des établissements d'enseignement et de recherche français ou étrangers, des laboratoires publics ou privés. 


\title{
Strain-induced crystallization in natural rubber:
} Flory's theory revisited

\author{
Paul Sotta ${ }^{* \dagger}$ and Pierre-Antoine Albouy ${ }^{\ddagger}$ \\ $\dagger$ †aboratoire Polymères et Matériaux Avancés, CNRS/Solvay, UMR5268, REII Centre \\ Lyon, 85 avenue des Frères Perret, 69192 Saint Fons Cedex, France \\ $\ddagger$ Laboratoire de Physique des Solides, CNRS/Université Paris-Sud, UMR8502, 91405 \\ Orsay Cedex, France \\ E-mail: paul.sotta-exterieur@solvay.com
}

\section{For Table of Contents use only}

Strain-induced crystallization in natural rubber: Flory's theory revisited P. Sotta, P.-A. Albouy
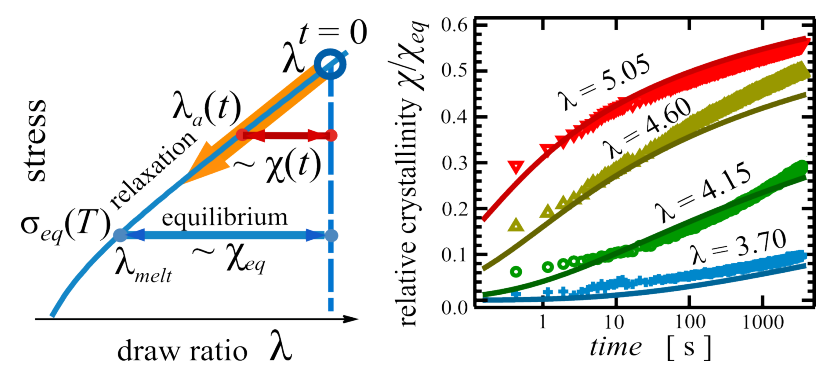


\begin{abstract}
In this paper, some aspects of the physical mechanisms involved in strain-induced crystallization (SIC) in cross-linked natural rubber networks are discussed. The theory of SIC as developed by Flory is considered within a somehow innovative perspective, in analogy to the liquid-gas phase transformation. A simple lever rule is proposed to relate the crystallinity index to the local and global draw ratios. Equilibrium properties are considered first. Some simple experiments are discussed within this framework in order to enlighten some fundamental aspects of SIC. As the order parameter for SIC is the draw ratio of the amorphous phase, the importance of directly measuring this parameter is emphasized. Special emphasis is put on the relaxation of the remaining amorphous fraction that accompanies SIC. The question of crystallization kinetics underlies most aspects of SIC. We show that the actual time-dependence of the crystalline content may be related to the mechanism of strain relaxation in a quite simple manner, using the lever rule mentionned above. Crystallization kinetics is also fundamental to explain the hysteretic behavior observed in dynamical conditions. Similarities and differences with static SIC are discussed. We transpose the classical theory of nucleation to the case of SIC and we discuss the kinetics within this framework.
\end{abstract}

\title{
1 Introduction
}

Strain-induced crystallization (SIC) in natural rubber (NR) has been reported ninety years ago $^{1}$ and the underlying physical mechanism was identified by Flory. ${ }^{2}$ In view of the high applicative importance of this material, it has been extensively studied since then. ${ }^{3-12}$ In particular, in-situ, real-time X-ray diffraction experiments have been developped in the last decade or so. ${ }^{13,14}$

Other theories have been proposed since the seminal work by Flory. ${ }^{15-20}$ While their predictions can be quantitatively different, they are generally based on quite similar physical ideas. Other approaches based on continuum mechanic approaches have been pro- 
posed. ${ }^{21-23}$ More recently, field theories treated in the Landau-Ginzburg formalism have been proposed. ${ }^{24,25}$ In Laghmach $2015,{ }^{24}$ the authors introduce an interfacial energy between the amorphous and crystalline phase (as in any classical nucleation theory) which is not constant but depends exponentially on the crystal size, in order to take into account the accumulation of topological constraints (crosslinks and entanglements) near crystal surfaces as crystals grow further. This prevents crystal growth beyond a typical size related to the density of topological constraints.

In this paper, we shall discuss some aspects of the physical mechanisms involved in SIC within a general thermodynamic point of view. In previous theoretical works, various assumptions on the detailed structure of the crystalline phase, as well as on the chain statistics and conformations, were made. ${ }^{2,15-17}$ While considering such details is certainly needed in order to obtain fully quantitative predictions, we show that the general framework of phase transformations considered here provides a semi-quantitative description of a bunch of observations. In fact, the profound analogy of the theory of SIC developed by Flory with e.g. the liquid-gas phase transformation of a substance, as implicitly noted already by Miyamoto et al., ${ }^{7}$ is emphasized. We believe it grasps the essential driving mechanisms at play. Particular emphasis will be put on the relaxation of the remaining amorphous fraction that accompanies SIC. Indeed, this phenomenon is likely to be so essential that the term crystallization-induced strain relaxation might perhaps be preferred to strain-induced crystallization in order to underline its importance.

The theory developed by Flory deals with thermodynamic equilibrium. However, in practice, the question of the crystallization kinetics underlies most aspects of SIC. A model for strain-induced crystallization kinetics based on a diffusion-limited process has been proposed. ${ }^{26,27}$

We discuss kinetic aspects still within a general thermodynamic framework. Emphasizing the underlying physics is essential to understand out-of-equilibrium and dynamical situations. We emphasize the similarities and differences between temperature-induced and 
strain-induced crystallization. We show that the actual time-dependence of the crystalline content may be related to the mechanism of strain relaxation in a quite simple manner. Crystallization kinetics is also fundamental to explain the hysteretic behavior observed in dynamical conditions. Similarities and differences with static SIC will be stressed.

What follows is restricted to the simple case of uniaxial deformation. Other geometries may be of interest for practical applications. ${ }^{28-30}$ The difficulty of their analysis essentially arises from the higher complexity of the associated strain field, while we claim the physics involved should remain essentially similar.

The paper is organized as follows. In Section 2 we first present the general thermodynamical framework which describes SIC at equilibrium. In Section 3 we then present the general framework to describe the kinetics of SIC. In Section 4 we illustrate the prediction of the theory by some selected experimental results from the literature. The simplifications, limitations and assumptions of the proposed framework are discussed in Section 5.

\section{Strain-induced crystallization at equilibrium}

Before considering strain-induced crystallization (SIC), it is useful to recall some very basic thermodynamics of phase transformations of a pure substance, as we claim SIC can be described within this framework, without considering at first detailed structural features of the crystalline phase. For practical illustration and for the sake of simplicity we consider the liquid-gas transition of a pure substance. ${ }^{31}$

\subsection{General thermodynamics of the liquid-gas transition}

The Gibbs free energy per molecule of a pure substance is a function $G(c, P, T)$ of the pressure $P$, temperature $T$ and an intensive variable $c$, defined here as the number density $c=1 / V$, where $V$ is the volume available per molecule. The variation $d G=-S d T+V d P$ is zero for constant $T$ and $P$, which insures that $T$ and $P$ are uniform at equilibrium. 
In some range of $T$ and $P$ values, $G$ considered as a function of $c$ (at fixed $T$ and $P$ ) has two minima which give the equilibrium values $c_{L}$ and $c_{G}$ of $c$ in the liquid (subscript $L$ ) and gas (subscript $G$ ) phases. $c_{G}(T, P)$ and $c_{L}(T, P)$ (or equivalently $P\left(V_{G}, T\right)$ and $\left.P\left(V_{L}, T\right)\right)$ are the constitutive equations and $G\left(c_{G}(T, P), P, T\right)$ and $G\left(c_{L}(T, P), P, T\right)$ the chemical potentials $\mu_{G}(T, P)$ and $\mu_{L}(T, P)$ of the gas and liquid phases respectively. For a given $P$ value, the temperature $T_{c}(P)$ at which $\mu_{G}=\mu_{L}$ is the equilibrium liquefaction temperature at pressure $P$. It obeys the standard ( $P$-dependent) relationship $T_{c}=Q / \Delta S_{f}$ in which $Q=\Delta H_{f}$ is the enthalpy of the transition. The curve $T_{c}(P)$ (or equivalently $P_{c}(T)$ ) defines the $(P, T)$ phase diagram.

A $(P, V, T)$ phase diagram can be drawn from the constitutive equations $P\left(V_{G}, T\right)$ and $P\left(V_{L}, T\right)$. A standard $P(V)$ isotherm (Clapeyron-Clausius) diagram is schematized in Figure 1. When the volume per molecule $V$ is decreased starting from the gas phase, the system at equilibrium at temperature $T$ follows the $D \longrightarrow C$ curve in gas phase until $P$ reaches the value $P_{c}(T)$, then the liquid-gas $C \longrightarrow B$ coexistence plateau, then the $B \longrightarrow A$ curve in the liquid phase.

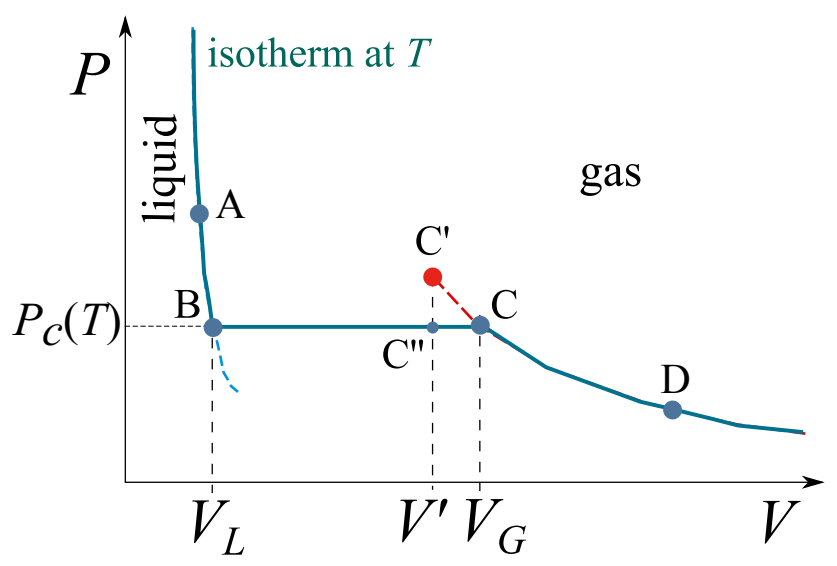

Figure 1: An equilibrium isotherm (blue curve) in the schematic $P(V)$ phase diagram for liquid gas transformation.

At pressure $P_{c}(T)$, any change of the volume of the system induces a change of the liquid fraction, meaning that phase transformation is induced by volume variation. If, starting from point $\mathrm{C}$ (pure gas), the volume is decreased down to $V^{\prime}$, or equivalently the gas pressure 
is increased to point C', the new equilibrium state will be point C", at pressure $P_{c}(T)$ and molar liquid fraction $\varphi_{L}$ given by the lever rule $\varphi_{L}=\left(V_{G}-V^{\prime}\right) /\left(V_{G}-V_{L}\right)$.

\subsection{Flory's theory of equilibrium strain-induced crystallization}

Figure 2 illustrates the different steps considered for the calculation of the thermodynamical changes occurring during SIC in Flory's theory. The behavior of the whole material is obtained by summing up the contributions of all chains (as schematized in Figure 2). As implicitly recognized by Miyamoto et al., ${ }^{7}$ this description has in fact deep analogy with the liquid-gas transition of a pure liquid. Working out this analogy enables recovering Flory's main conclusions in a simple way. Flory's theory essentially relies on the following assumptions for chain conformations: (1) Chains within crystallites are perfectly oriented along the stretching axis and chain statistics in directions perpendicular to the stretching axis are not affected by crystallization, apart perhaps from the shortening of the amorphous sub-chain; (2) Chains do not re-enter a crystallite by folding; (3) Chain elongation in the amorphous phase remains moderate so that Gaussian statistics apply. The possibility of chain folding and extension to non-Gaussian statistics have been considered by other authors. ${ }^{16}$ In a recent work the authors consider the additional loss of entropy associated to the constraint that some points in the middle of a chain are fixed at crystal surfaces. ${ }^{32}$ This effect would however induce an increase of the stress at onset of SIC, that is not observed.

In the following a one-dimensional simple model, which fully parallels the gas-liquid transition, is outlined. The above hypotheses are implicit in this thermodynamic model. No detailed description of the crystal shape and structure is needed at this stage. Under uniaxial tensile strain, the variation of the energy is $d U=T d S+\sigma d \lambda$. The first term $d Q=T d S$ is the heat variation, the second term is the work provided by the stress $\sigma$ when the stretching ratio $\lambda=L / L_{0}$ varies from $\lambda$ to $\lambda+d \lambda$. The term $P d V$ related to the work of hydrostatic pressure is neglected, assuming that volume variation at crystallization is neglected (then it does not matter to consider the energy $U$ instead of the enthalpy $H$ ). 


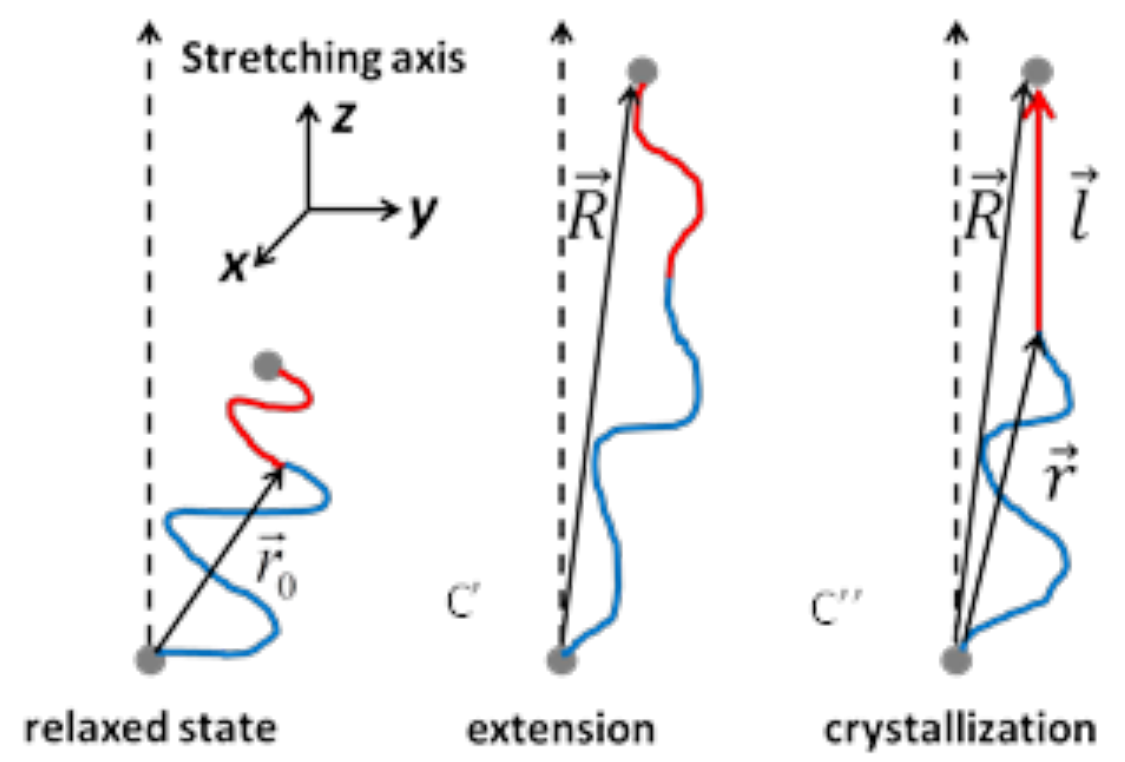

Figure 2: Schematics of the crystallization process in Flory's theory. Crystallizing part of a extended chain relaxes the extension degree of the remaining amorphous part. This is the driving force of strain-induced crystallization.

Also, as the considered problem is one-dimensional, the conserved quantity should be the tensile force, i.e. the engineering stress. Accordingly, in what follows, we shall use the relationship between the engineering stress and the draw ratio as the constitutive equation of the amorphous fraction. As proposed by Miyamoto et al, ${ }^{7}$ the appropriate thermodynamic potential to consider for uniaxial elongation is (per unit volume of polymer):

$$
\Omega=U-T S-\sigma \lambda
$$

The variation $d \Omega=-S d T-\lambda d \sigma$ is zero for constant $T$ and $\sigma$, which insures that the temperature $T$ and stress $\sigma$ (and of course implicitly the hydrostatic pressure) are the intensive variables which must be uniform within each phase at equilibrium. The generalized thermodynamic potential is a function $\Omega_{N}(y, \sigma, T)$, where $y$ is an intensive variable, the so-called "order parameter", which should discriminate the different phases, in analogy with the volume per molecule in the liquid-gas transition. Subscript $N$ indicates that, for a crosslinked system (an elastomer), there is a dependence on the average number $N$ of statistical segments 
between crosslinks and/or entanglements, related to the number density of elastic chains $\nu$ (for a tetrafunctional network, this is twice the crosslink density) by $\nu \approx 1 / N a^{3}$, where $a^{3}$ is the volume per statistical segment.

The draw ratio $\lambda$ is the simplest, natural choice for the order parameter $y$. At the scale of a single chain, the "natural" average length of a chain along the stretching direction in the amorphous (random coil) configuration may be defined by $\left\langle z^{2}\right\rangle_{0}{ }^{1 / 2}=a(N / 3)^{1 / 2}$. In a hypothetical fully crystalline state (subscript $c$ ), the chain would be fully stretched along the stretching direction: $\left\langle z^{2}\right\rangle_{c}{ }^{1 / 2}=a N$, which would correspond to an effective elongation ratio

$$
\lambda_{c}=\frac{\left\langle z^{2}\right\rangle_{c}{ }^{1 / 2}}{\left\langle z^{2}\right\rangle_{0}{ }^{1 / 2}}=(3 N)^{1 / 2}
$$

The parameter $\lambda_{c}$ shall play a key role in what follows. As $N>>1, \lambda_{c}$ should be significantly larger than one while, according to hypothesis (3) above, one should assume the elongation ratio of the amorphous phase $\lambda_{a}<<(3 N)^{1 / 2}$.

In some range of $T$ and $\sigma$ values, $\Omega_{N}(\lambda, \sigma, T)$ has two minima which define the potentials $\Omega_{c}$ and $\Omega_{a}$ of the crystalline and amorphous phases, respectively. Within this range, for each value of $\sigma$, there is a temperature $T_{m}(\sigma)$, or equivalently a value $\sigma_{e q}(T)$ for a given temperature $T$, such that $\Omega_{c}=\Omega_{a}$. The $(\sigma, T)$ phase diagram is given by the way in which $T_{m}(\sigma)$ varies as a function of $\sigma$, or equivalently, $\sigma_{e q}(T)$ varies as a function of $T$. Then, the locations $\lambda_{c}(\sigma)$ and $\lambda_{a}(\sigma)$ of the minima of $\Omega_{N}(\lambda, \sigma, T)$ for each value of $T$ are the equilibrium constitutive equations of the coexisting phases. The $\lambda(\sigma)$, or equivalently $\sigma(\lambda)$, diagram is equivalent to the liquid-gas Clapeyron-Clausius diagram. $\sigma_{e q}(T)$ defines the stress at equilibrium melting at temperature $T$. Equivalently, the corresponding draw ratio $\lambda_{\text {melt }}$ in the amorphous phase is the draw ratio at equilibrium melting at $T$.

An explicit expression for the thermodynamic potential $\Omega_{N}(\lambda, \sigma, T)$ is not known a priori. In the amorphous phase, the thermodynamic potential (per unit volume) is given by rubber elasticity theory, which, in its simplest form, for a tetrafunctional, affine network, may be 
written: ${ }^{33,34}$

$$
\Omega_{a}=\frac{k_{B} T \nu}{2}\left(\lambda^{2}+2 \lambda^{-1}-3\right)-\sigma \lambda
$$

The reference $\Omega_{a}=0$ was taken in the relaxed state. Using the constitutive equation of the amorphous (elastomeric) phase ${ }^{33,34}$

$$
\sigma=k_{B} T \nu\left(\lambda-\lambda^{-2}\right)
$$

$\Omega_{a}$ can be expressed as a function of $\lambda$ :

$$
\Omega_{a}=-\frac{k_{B} T \nu}{2}\left(\lambda^{2}-4 \lambda^{-1}+3\right)
$$

By inverting Equation (4), $\lambda$ may in principle be expressed as a function of the stress $\sigma$ and $\Omega_{a}$ in Equation (3) may be expressed as a function of $\sigma$. Assuming $\lambda^{3}>>1$ at SIC onset, the linear approximation $\sigma \approx k_{B} T \nu \lambda$ can be used in Equation (4) and an approximate expression for $\Omega_{a}$ as a function of $\sigma$ in the vicinity of SIC onset is then

$$
\Omega_{a} \approx-\frac{k_{B} T \nu}{2}\left(\frac{\sigma^{2}}{\left(k_{B} T \nu\right)^{2}}+3\right)=-\frac{k_{B} T}{2 N a^{3}}\left(\frac{\left(N a^{3}\right)^{2} \sigma^{2}}{\left(k_{B} T\right)^{2}}+3\right)
$$

The constitutive law Equation (3) corresponds to the simplest case of an incompressible, hyperelastic (Neo-Hookean) material.

In the crystalline phase, the thermodynamic potential may be written in the standard form (still refered to the relaxed state):

$$
\Omega_{c}=-Q\left(\frac{T_{m}^{*}-T}{T_{m}^{*}}\right)-\sigma \lambda_{c}
$$

where $Q$ is the enthalpy of melting per unit volume, $T_{m}^{*}$ the melting temperature in the relaxed state, and $T>T_{m}^{*}$. Note that the entropy of the crystalline phase, which is not strictly zero due to some conformational disorder, is implicitly taken into account in $\Omega_{c}{ }^{35}$ 
The thermodynamic potentials $\Omega_{c}(\sigma, T)$ and $\Omega_{a}(\sigma, T)$, as well as the curve $\lambda_{\text {melt }}(T)$ resulting from the equilibrium condition $\Omega_{c}=\Omega_{a}$, are schematized in Figure 3.

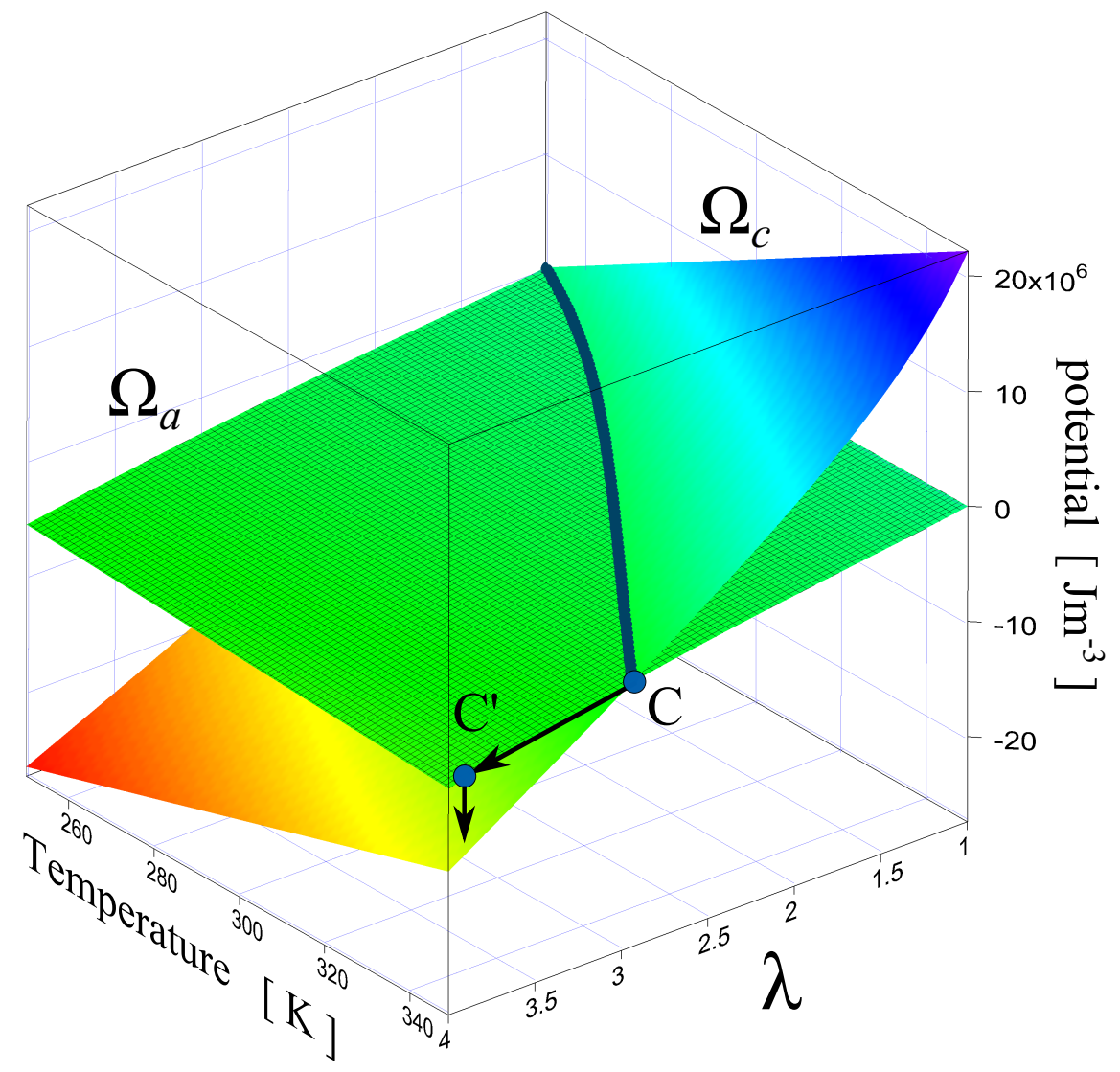

Figure 3: The thermodynamic potentials $\Omega_{c}(\lambda, T)$ and $\Omega_{a}(\lambda, T)$ per unit volume, computed from equations (3) and (7), with $N=40, Q=4.7 \mathrm{~kJ} / \mathrm{mol}$ and $T_{m}^{*}=250 \mathrm{~K} . \lambda$ is the stretching ratio. The thick black curve corresponds to the curve $\lambda_{\text {melt }}(T)$ resulting from the equilibrium condition $\Omega_{c}=\Omega_{a}$ (see Equation 14). Point $\mathrm{C}$ corresponds to the stretching ratio $\lambda_{\text {melt }}$ at equilibrium melting at temperature $T$. Point C' corresponds to a metastable, overstretched system (see Section 3.2).

In the crystalline phase, the constitutive equation is of the form $\Delta \sigma=G_{c} \Delta \lambda$ where $G_{c}$ is the Young's modulus of the crystalline phase. $G_{c}$ is a decreasing function of temperature and is of order $10^{9} \mathrm{~Pa}$, much higher than the modulus of the amorphous phase, of order 0.5 MPa typically.

$\sigma(\lambda)$ isotherms (that is, a Clapeyron-Clausius diagram) can be established on this basis, as schematized in Figure 4. Starting from an amorphous system (point D in Figure 4), the system first follows path $D \longrightarrow C$ along the constitutive equation of the amorphous phase as 
the engineering stress is progressively increased. The system enters the coexistence plateau at point $\mathrm{C}$, which corresponds to the equilibrium value $\sigma_{e q}(T)$. For a given value of the macroscopic stretching ratio $\lambda$, the equilibrium crystalline fraction $\chi$ is given by the lever rule:

$$
\chi=\frac{\lambda-\lambda_{a}}{\lambda_{c}-\lambda_{a}}
$$

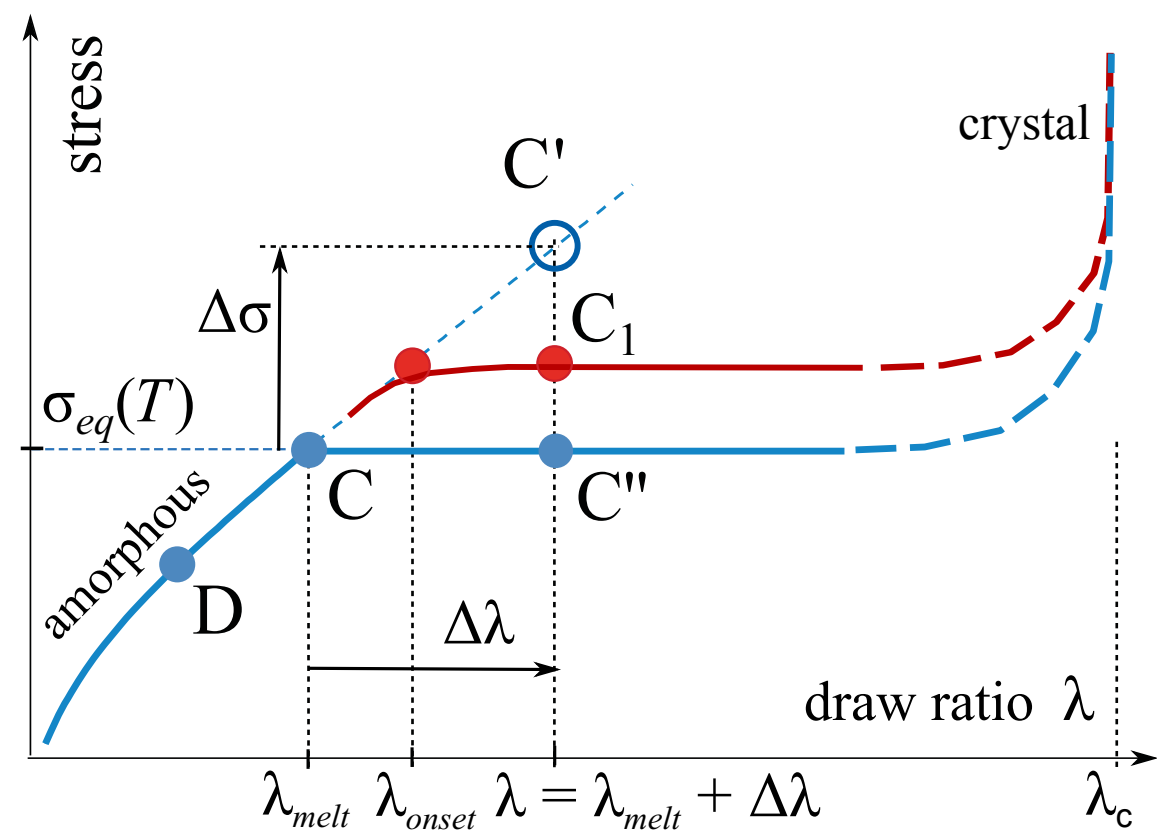

Figure 4: $\sigma(\lambda)$ 'Clapeyron-Clausius' diagram in a uniaxially stretched NR elastomer. The blue curve is an equilibrium isotherm. $\sigma_{e q}(T)$ is the stress at amorphous/crystal coexistence at temperature $T$. When the amorphous material (stretching ratio $\lambda_{\text {melt }}$, point $\mathrm{C}$ ) is overstretched up to $\lambda=\lambda_{\text {melt }}+\Delta \lambda$ (C to C'), it relaxes from point C' down to C". At C", the stretching ratio of the amorphous phase is $\lambda_{\text {melt }}$ and the crystallinity index is given by the lever rule Equation (8) with $\lambda_{a}=\lambda_{\text {melt }}$. However, due to kinetic limitation, relaxation within a limited amount of time reaches point $\mathrm{C}_{1}$ (instead of $\mathrm{C}^{\prime \prime}$ ), with the amorphous phase strained to a value $\lambda_{\text {onset }}>\lambda_{\text {melt }}$ and the crystallinity shall be given by the lever rule on the red plateau with $\lambda_{a}=\lambda_{\text {onset }}$. On stretching at a finite rate, crystallization starts at $\lambda_{\text {onset }}>\lambda_{\text {melt }}$.

This lever rule is at the core of the model and plays a key role in what follows. It is simply based on the geometrical conservation of the overall sample length throughout the crystallization process. It thus applies both to equilibrium and out-of-equilibrium situations. It has been demonstrated experimentally by a series of careful measurements of the draw 
ratio of the amorphous phase as SIC proceeds, as will be discussed in Section 4. ${ }^{36,37}$ Any change of the macroscopic elongation $\lambda$ of the sample induces a change of the crystalline fraction. In that sense, it may be said that the phase transformation is induced by the variation in stretching ratio, or equivalently, that the driving force for crystallization is overstretching of the amorphous phase. The decrease of stress associated to crystallization (from point C' down to the equilibrium point C" in Figure 4) can be computed from equation (8). Rewriting Equation (8) as:

$$
\lambda_{a}=\frac{\lambda-\chi \lambda_{c}}{1-\chi}
$$

approximating $\sigma \approx k_{B} T \nu \lambda_{a}$ and using equation (2) gives:

$$
\sigma \approx \frac{k_{B} T \nu}{1-\chi}\left(\lambda-\chi(3 N)^{1 / 2}\right)
$$

Equation (10) is in fact very similar to Flory's formula (within the same level of approximation $\left.1 / \lambda^{2}<<\lambda\right)$ :

$$
\sigma \approx \frac{k_{B} T \nu}{1-\chi}\left(\lambda-\chi\left(\frac{6 N}{\pi}\right)^{1 / 2}\right)
$$

with $\lambda_{c}=(3 N)^{1 / 2}$ instead of $(6 N / \pi)^{1 / 2}$ in the original Flory's work.

The dependence of the equilibrium melting temperature $T_{m}(\lambda)$ on $\lambda$ can easily be deduced as well. At equilibrium melting, the potentials $\Omega_{c}$ and $\Omega_{a}$ are equal, which gives directly the melting temperature as a function of the engineering stress $\sigma$ and melting enthalpy $Q$ (assumed to be independent of $T$ and $\lambda$ ). Combining equations (3) and (7) gives:

$$
\frac{1}{T_{m}^{*}}-\frac{1}{T_{m}(\lambda)}=\frac{R}{N Q}\left[\lambda \lambda_{c}-\frac{\lambda^{2}}{2}+\frac{2}{\lambda}-\frac{\lambda_{c}}{\lambda^{2}}-\frac{3}{2}\right]
$$

where $R$ is the gas constant. Considering that $\lambda_{c}=(3 N)^{1 / 2}$ and restricting to most significant factors at high elongation, equation (12) is again quite similar to the original 
Flory's equation:

$$
\frac{1}{T_{m}^{*}}-\frac{1}{T_{m}(\lambda)}=\frac{R}{Q}\left[\lambda\left(\frac{6}{\pi N}\right)^{1 / 2}-\frac{1}{N}\left(\frac{\lambda^{2}}{2}+\frac{1}{\lambda}\right)\right]
$$

Note that, in contrast to the original Flory's equation (13), equation (12) can be extrapolated to $\lambda=1$.

Equivalently, Eq. (12) can be used to express the value $\lambda_{\text {melt }}(T)$ at a given temperature $T:$

$$
\frac{1}{T_{m}^{*}}-\frac{1}{T}=\frac{R}{N Q}\left[\lambda_{m e l t} \lambda_{c}-\frac{\lambda_{m e l t}^{2}}{2}+\frac{2}{\lambda_{m e l t}}-\frac{\lambda_{c}}{\lambda_{m e l t}{ }^{2}}-\frac{3}{2}\right]
$$

The energy balance along the path from $\mathrm{C}$ up to C' then to C" in Figure 4 can be delineated as follows:

- from $\mathrm{C}$ to $\mathrm{C}$ ': according to entropic rubber elasticity, the work provided to stretch the sample is transformed into entropy decrease, i.e. it is transferred into heat generated into the sample, while internal energy is not affected. This statement, however, would be correct for a fully entropic system only. In practice, there generally exists a small enthalpic change upon stretching (about $10 \%$ of the stored mechanical work). ${ }^{34}$ For an adiabatic process, sample temperature increases. Recent quantitative calorimetric approaches have been proposed to measure SIC based on this energy balance. ${ }^{38,39}$

- from C' to C": two changes occur concomitantly. First, a fraction of the material crystallizes, which generates further heat in the sample. Second, the strain in the amorphous part relaxes from $\lambda_{\text {melt }}+\Delta \lambda$ down to $\lambda_{\text {melt }}$, which increases entropy and thus absorbs heat.

The above description of equilibrium crystallization can be summarized by a 3D (Clapeyron-Clausius) diagram as shown in Figure 5. The stress $\sigma$ as a function of $T$ and $\lambda$ is schematized as a surface with three different parts. The first part, below the equilibrium melting curve (thick black curve) corresponds to the hyperelastic constitutive law of the amorphous phase, the second part corresponds to the coexistence plateau and the third part 
to the crystalline phase. As the modulus of the crystal phase is much larger than that of the amorphous phase, this part of the 3D surface is drawn nearly vertical. Note that the extension of a DNA molecule as described in ${ }^{40}$ also corresponds exactly to this model.

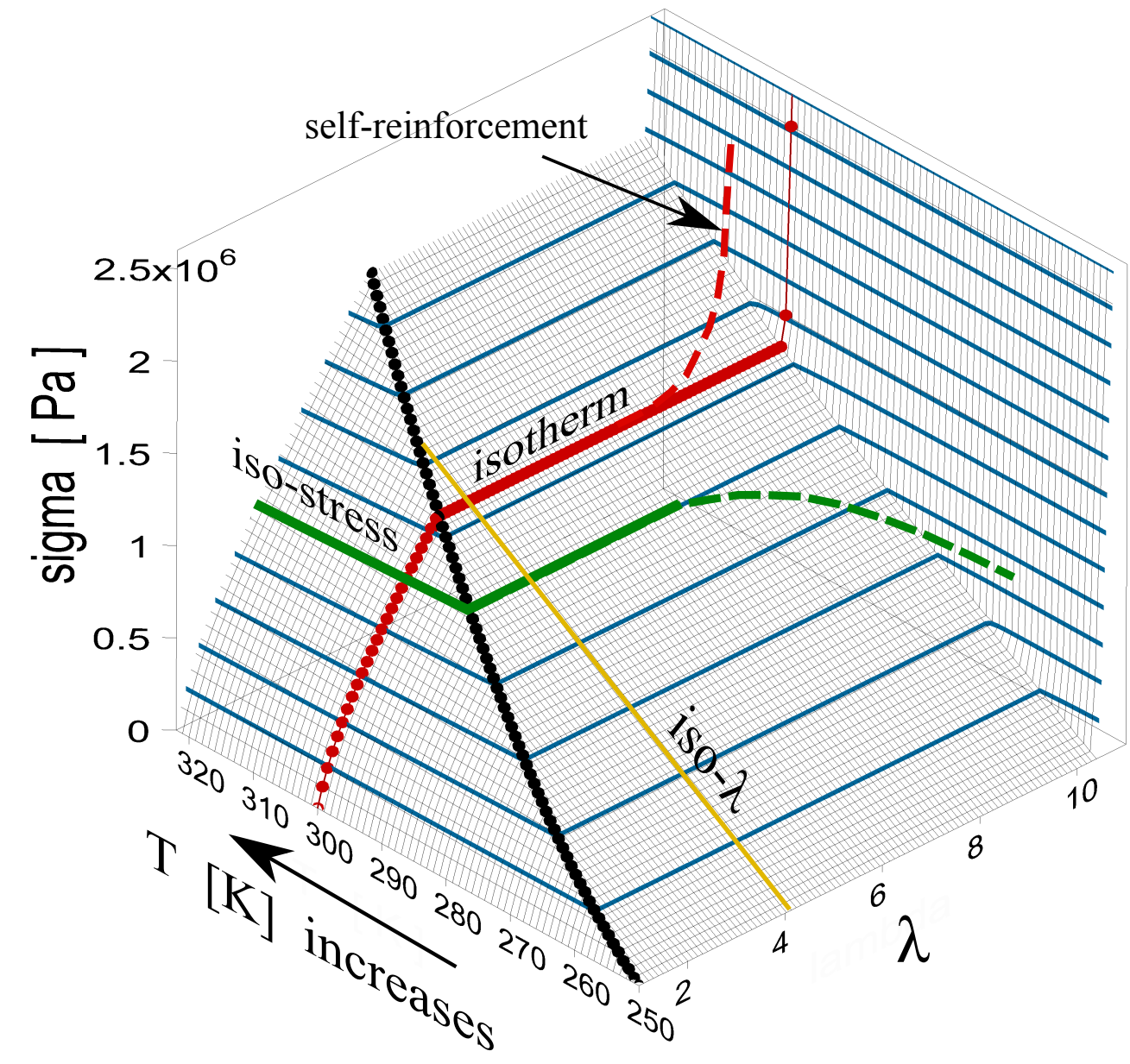

Figure 5: Three-dimensional $\sigma(\lambda, T)$ (Clapeyron-Clausius) diagram for the equilibrium between amorphous and crystalline phases in a uniaxially stretched NR elastomer. Figure 4 corresponds to a slice along an isotherm (red curve). Experiments by Miyamoto et al. correspond to iso-stress curves (green horizontal curve). ${ }^{7}$ Iso- $\lambda$ curves as a function of temperature (yellow curve) correspond to equilibrium crystallization curves shown e.g. by Trabelsi et al. ${ }^{6}$

\section{Kinetics}

Polymer crystallization in general and specifically SIC is a kinetic phenomenon which can extend to very long times scales, over orders of magnitude. ${ }^{41,42}$ Actually in most experimental 
circumstances, measured SIC parameters are driven by kinetic phenomena rather than by the equilibrium equations. These kinetic aspects of SIC can be put into evidence in various ways. The first way is to draw samples at various, fixed draw rates, and investigate how the onset of SIC depends on the draw rate and on temperature (path $\mathrm{C}$ to $\mathrm{C}$ ' in Figure 4). A second way is to draw samples in a quasi-instantaneous way at various, fixed draw ratios beyond the SIC onset, and investigate the subsequent time evolution of the crystalline ratio, as a function of the imposed draw ratio (or more precisely, of the over-stretching) or of temperature (path C' to C"' in Figure 4).

\subsection{Thermal crystallization}

In contrast to SIC, the kinetics of temperature-induced crystallization of polymers have been extensively studied. ${ }^{41,43-45}$ Some partial basic results are summarized here, as the kinetics of SIC has a number of features which parallel those of thermally induced crystallization. The driving force for thermal crystallization is the supercooling $\Delta T=T_{m}-T$, where $T_{m}$ is the equilibrium melting temperature. The kinetics is driven by nucleation and growth mechanisms. ${ }^{46}$ Nucleation can be homogeneous or, in most practical cases, heterogeneous, related to the presence of impurities or heterogeneities acting as nucleation centers.

For a polymer confined in a set of small cells of volume $v$ such as microdroplets ${ }^{47}$ or confined within a micro- or nanotemplate, ${ }^{48}$ nucleation alone is the relevant mechanism. In this case the evolution of the crystallinity index $\chi(t)$ during isothermal crystallization is described by

$$
\chi(t)=1-\exp [-v \omega t] \approx v \omega t
$$

where $\omega$ is the nucleation rate per unit volume of amorphous polymer, with $v \omega t<<1$. It was assumed in writing Equation (15) that only one nucleus is formed at random within each cell, which fills the volume $v$ of the cell very fast compared to the overall crystallization 
kinetics and stops further growth. It is further assumed that nucleation is unaffected by the presence of surfaces or interfaces and that the nucleation rate $\omega$ is constant throughout the evolution, i.e. so-called athermal effects are neglected. ${ }^{49}$ The nucleation rate depends on temperature and is expressed by the general expression:

$$
\omega=\omega_{0} \exp \left[-\frac{B}{k_{B} T}\right]
$$

where the nucleation barrier $B$ (the free energy barrier to produce a nucleus of critical size) is for a cylindrical crystal ${ }^{31}$

$$
B=\frac{8 \pi \gamma_{s}^{2} \gamma_{e} T_{m}{ }^{2}}{Q^{2}(\Delta T)^{2}}
$$

where $\gamma_{s}$ and $\gamma_{e}$ are the side and end crystal surface energies respectively, $T_{m}$ is the equilibrium melting temperature and $Q$ is the melting enthalpy. ${ }^{47} \mathrm{~A}$ slightly different expression was proposed by Hoffman to take the temperature variation of $Q$ into account. ${ }^{50}$

Equations (15) to (17) describe isothermal crystallization. Often crystallization is recorded during a cooling ramp at a given cooling rate $r$. Infering an effective time-temperature superposition principle by relating the evolution of the crystallinity index during a cooling ramp to isothermal equations above is not straigthforward and relies on several assumptions, such as the so-called 'additivity' principle, which states that the rate of crystallization at any temperature depends only on this temperature and not on prior thermal history. ${ }^{51}$ In this case, it follows from Equation 15 that, if the cooling rate $r$ and temperature $T$ change concomitantly in such a way that the quantity $\omega(T) / r$ remains constant, then a master curve should in principle be obtained.

The above considerations apply when the crystallinity index remains small, typically smaller than 0.3 , i.e. relatively close to the onset of crystallization. At further stages of crystallization, impingement must be considered and the equations describing time evolution become more complex. ${ }^{52}$ 


\section{$3.2 \quad$ Nucleation barrier for SIC}

In what follows we shall assume that crystallization is kinetically limited by homogeneous nucleation, as it was similarly assumed by Candau et al. ${ }^{53}$ In crosslinked materials such as Natural Rubber and other elastomers, the fact that nucleation is the limiting kinetic factor is justified by the small size of crystallites. Growth beyond nanometer sizes is impeded by the presence of crosslinks. In classical nucleation theory, crystal nucleation occurs within a metastable (either supercooled or over-stretched) system because creating a nucleus of the thermodynamically stable (crystal) phase generates an interface which has an energy cost.

At a temperature $T$, an amorphous NR sample (point $\mathrm{C}$ in Figure 6, see also Figures 3 and 4 ), is instantateously stretched to and maintained at a fixed extension ratio $\lambda$ corresponding to the metastable point $\mathrm{C}^{\prime}$, that is, from $\sigma_{e q}(T)$ to $\sigma_{e q}(T)+\Delta \sigma$ (Figure 4 ) or equivalently from $\lambda_{\text {melt }}$ to $\lambda=\lambda_{\text {melt }}+\Delta \lambda$. It shall then relax towards equilibrium at point C", $\lambda$ being kept fixed. To keep things simple, a cylindrical crystal (height $h$, radius $\xi$ ) is considered. The variation of free energy $\Delta H$ when a crystal is formed includes surface terms and a volume term: ${ }^{24,31}$

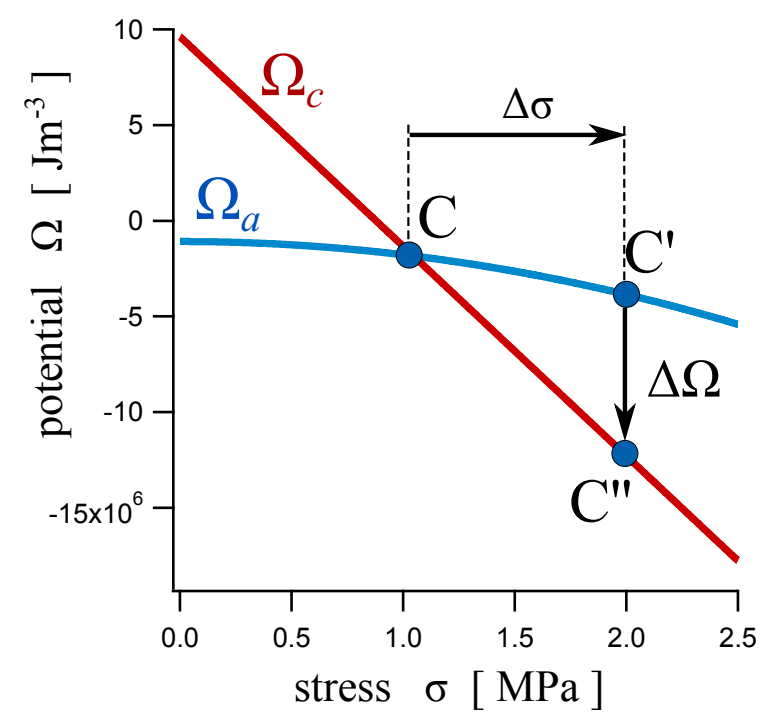

Figure 6: The thermodynamic potentials $\Omega_{a}$ and $\Omega_{c}$ as a function of stress $\sigma$ at $T=293 \mathrm{~K}$ (see also Figure 3. The sample is stretched from the equilibrium melting point $\mathrm{C}$ up to point C' and the material within a crystalline nucleus then relaxes down to point C'. 


$$
\Delta H=2 \pi \xi h \gamma_{s}+2 \pi \xi^{2} \gamma_{e}-\pi \xi^{2} h \Delta \Omega
$$

The critical nucleation sizes $h_{c}$ and $\xi_{c}$ given by the conditions $\partial(\Delta H) / \partial \xi=0$ and $\partial(\Delta H) / \partial h=0$ are

$$
\xi_{c}=\frac{2 \gamma_{s}}{\Delta \Omega} ; \quad h_{c}=\frac{4 \gamma_{e}}{\Delta \Omega}
$$

The variation of the volume term $\Delta \Omega=\Omega\left(C^{\prime}\right)-\Omega\left(C^{\prime \prime}\right)>0$ is the work done when moving the interface. It is estimated to be (see Figure 6):

$$
\Delta \Omega=\left(\Omega\left(C^{\prime}\right)-\Omega(C)\right)-\left(\Omega\left(C^{\prime \prime}\right)-\Omega(C)\right)=\lambda_{c} \Delta \sigma-\frac{\partial \Omega_{a}}{\partial \sigma} \Delta \sigma
$$

where the thermodynamic potential in the amorphous phase is given by equation (3), with $\lambda$ given as a function of $\sigma$ through inverting equation (4). From equation (4) one has directly $\partial \Omega_{a} / \partial \sigma \approx \lambda_{a}$, where $\lambda_{a}$ is the instantaneous (time-dependent) draw ratio of the amorphous phase. Equation (20) above may thus be rewritten:

$$
\Delta \Omega=\Delta \sigma\left(\lambda_{c}-\lambda_{a}\right)
$$

which is completely analogous to the equivalent equation for the liquid-gas transition $\Delta \mu=\left(v_{G}-v_{L}\right) \Delta P$. Finally the nucleation barrier to crystallize is:

$$
B=\Delta H\left(\xi_{c}\right)=\frac{8 \pi \gamma_{s}^{2} \gamma_{e}}{\Delta \sigma^{2}\left(\lambda_{c}-\lambda_{a}\right)^{2}}
$$

$\lambda_{a}$ is equal to the imposed $\lambda$ at $t=0$. When considering the factor $\lambda_{c}-\lambda_{a}$, the difference between $\lambda_{a}$ and $\lambda$ should not be important, as $\lambda_{c}$ is significantly larger than $\lambda$. The driving force is the $\Delta \sigma^{2}$ factor, which depends on the over-stretching parameter $\Delta \lambda=\lambda_{a}(t)-$ $\lambda_{\text {melt }}$. Using the approximate constitutive equation $\Delta \sigma=k_{B} T \nu \Delta \lambda=k_{B} T \Delta \lambda /\left(N a^{3}\right)$, the nucleation barrier may be equivalently rewritten: 


$$
B=\frac{8 \pi N^{2} a^{6} \gamma_{s}^{2} \gamma_{e}}{\left(\lambda_{c}-\lambda_{a}\right)^{2}\left(k_{B} T\right)^{2} \Delta \lambda^{2}}
$$

which again is analogous to the standard expression for the nucleation barrier. ${ }^{31,47,50}$

\subsection{Kinetic equation for crystallization}

In all what follows, it shall be assumed that the lever rule Equation (8) remains valid in outof-equilibrium situations, based on the hypothesis that stress relaxation in the amorphous phase is extremely fast with respect to typical time scales involved in stretching experiments.

SIC experiments can be performed by overstretching the sample to a constant extension ratio $\lambda$ and recording the time evolution of the crystallinity index, which is somehow analogous to isothermal crystallization. There is however a notable difference. According to the

lever rule Equation (8), the local over-stretching of the amorphous phase, which provides the driving force for SIC, relaxes down as SIC proceeds during an experiment at constant $\lambda$, while during isothermal crystallization, the supercooling $\Delta T$ stays constant. $\lambda_{a}$ might in principle relax down to $\lambda_{\text {melt }}$ if true equilibrium would be reached. It follows that the nucleation rate shall not be constant, contrary to the case of isothermal crystallization. We neglect so-called athermal effects, meaning that we assume that the nucleation rate depends on the instantaneous degree of over-stretching only, not on the whole crystallization history.

In a relaxation experiment in which the overall draw ratio $\lambda$ of the sample is maintained fixed $(d \lambda / d t=0)$, after a time $t$, the system has reached a crystalline fraction $\chi(t)$ and the stress has relaxed down to a value $\sigma(t)$ (see point $\mathrm{C}_{1}$ in Figure 4). Accordingly the draw ratio of the amorphous phase has relaxed from the initial value $\lambda$ to a value $\lambda_{a}(t)$, related to $\chi(t)$ by the lever rule Equation (8).

Based on Equation (22), the nucleation barrier at time $t$ is:

$$
B(t)=\frac{8 \pi \gamma_{s}^{2} \gamma_{e}}{\Delta \sigma^{2}(t)\left(\lambda_{c}-\lambda_{a}(t)\right)^{2}}
$$


and the crystallization rate $\omega$ per unit volume at time $t$ is given by:

$$
\omega=f_{0} \exp \left[-\frac{H(t)}{k_{B} T}\right]
$$

where $f_{0}$ is the nucleation frequency per unit volume. The kinetic equation for crystallization is then

$$
\frac{1}{1-\chi} \frac{d \chi}{d t}=\omega(t)
$$

The nucleation barrier Equation (24) can be rewritten (in $k_{B} T$ units):

$$
\frac{B(t)}{k_{B} T}=\frac{b_{0}(1-\chi)^{4}}{\left(1-\chi_{e q}\right)^{2}\left(\chi_{e q}-\chi\right)^{2}}
$$

in which $b_{0}$ is the quantity

$$
b_{0}=\frac{g_{0}}{\left(\lambda_{c}-\lambda_{\text {melt }}\right)^{4}}
$$

with $g_{0}$ the factor

$$
g_{0}=\frac{8 \pi \gamma_{s}^{2} \gamma_{e}}{k_{B} T G^{2}}
$$

The quantity $\Delta \sigma$ in equation 24 has been replaced by $\Delta \sigma=G \Delta \lambda=G\left(\lambda-\lambda_{\text {melt }}\right)$, where $G$ is the modulus of the amorphous (elastomer) phase, and the lever rule Eq. (8) was used.

Altogether Equation (26) can then be rewritten as an explicit equation for the kinetic evolution of $\chi(t)$ :

$$
\frac{1}{1-\chi} \frac{d \chi}{d t}=f_{0} \exp \left[-\frac{b_{0}(1-\chi)^{4}}{\left(1-\chi_{e q}\right)^{2}\left(\chi_{e q}-\chi\right)^{2}}\right]
$$

The general behavior described by Equation (30) is illustrated in Figures 7 and 8. For $\gamma_{s} \approx \gamma_{e} \approx 10^{-2} \mathrm{~N} / \mathrm{m}, k_{B} T \approx 4 \times 10^{-21} \mathrm{~J}\left(T=20^{\circ} \mathrm{C}\right), G \approx 0.5 \times 10^{6} \mathrm{~Pa}, \lambda_{c} \approx 2.6$ and 
$\lambda_{\text {melt }}=14.8$, the factor $b_{0}$ is of order a few $10^{-1}$.

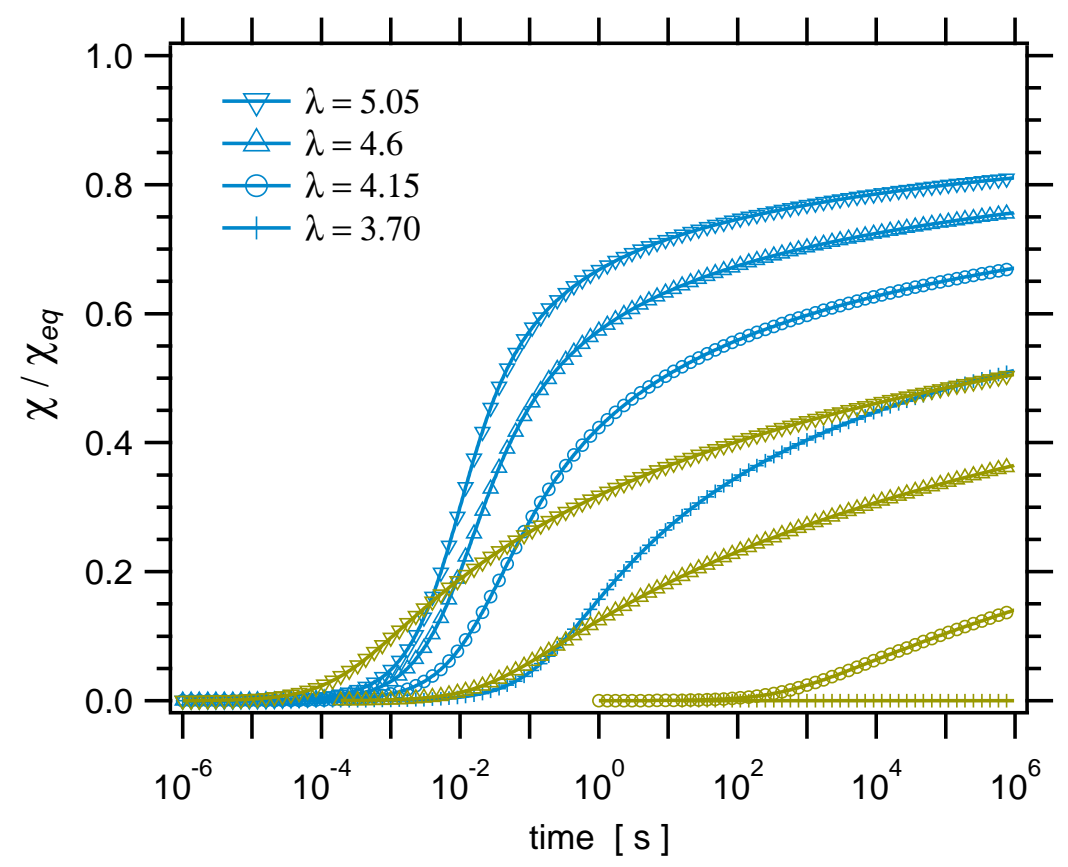

Figure 7: The crystallization kinetics curves $\chi / \chi_{e q}$ obtained by solving Equation (30) at different stretching ratios $\lambda$ as indicated in the legend and two different sets of parameters: Blue curves: prefactor $f_{0}=61$; factor $g_{0}=10^{3}$, corresponding to an initial energy barrier $b_{0}=0.0456$; Green curves: prefactor $f_{0}=10^{8}$; factor $g_{0}=8 \times 10^{3}$, energy barrier $b_{0}=0.365$.

The barrier height $b_{0}$ drives the sensitivity of the curves to the applied stretching ratio $\lambda$. The higher $b_{0}$, the more pronounced is the dependence of the curves and of the crystallization onset on $\lambda$. The sets of curves shown in Figure 7 were obtained with $g_{0}=10^{3}$ and $g_{0}=8 \times 10^{3}$, corresponding respectively to surface energies $\gamma \approx 3 \times 10^{-3}$ and $\gamma \approx 10^{-2} \mathrm{~N}^{-1}$ (in the hypothesis $\gamma_{e} \approx \gamma_{s} \approx \gamma$ ).

Would the nucleation barrier $B$, or equivalently the rate $\omega$, be constant along the kinetic evolution, then a $\lambda$-vs-time superposition principle could be applied to the set of curves shown in Figure 7. It would be possible to change both the time scale and the value of $\lambda$ in such a way that the product $\omega t$ remains constant, which would lead to a master curve for $\chi(t)$. The evolution of each set of curves is however more complex and $\lambda$-vs-time superposition does not work in an exact way. However, it may be inferred from e.g. the set of green curves in Figure 7, that such superposition for the $\chi / \chi_{e q}$ curves may be approximately true. 


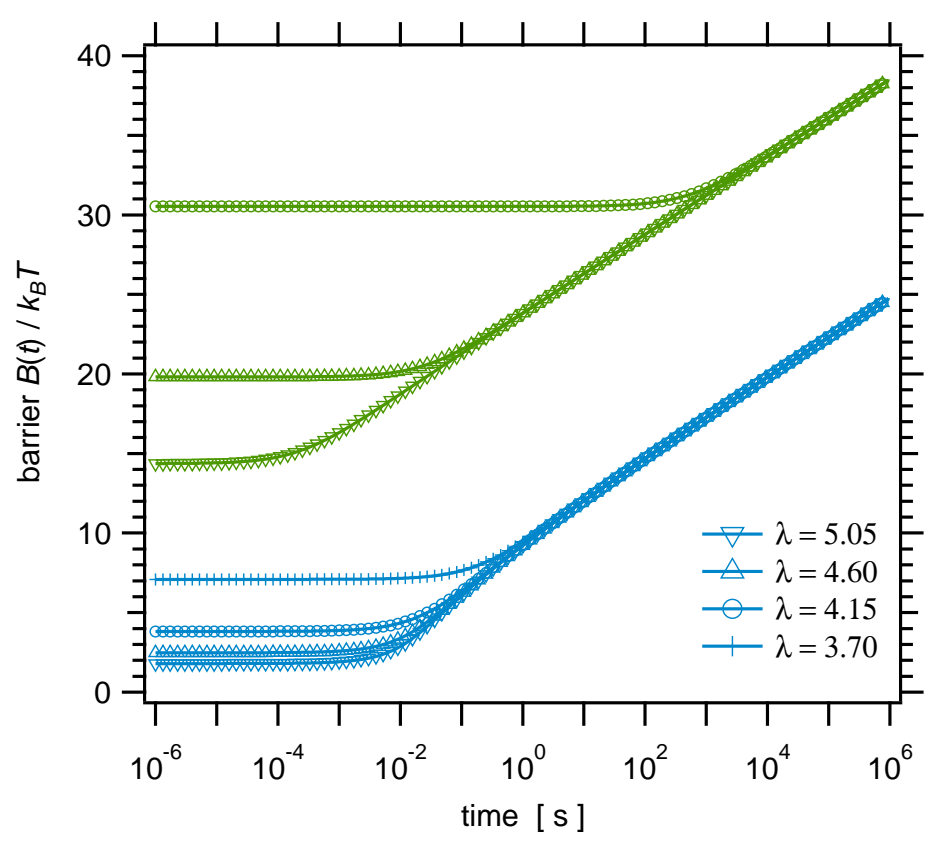

Figure 8: The nucleation barrier $B(t)$, Equation (24) or (27) as a function of time at different stretching ratios $\lambda$ as indicated in the legend and two different sets of parameters: blue curves: prefactor $v f_{0}=61$; factor $g_{0}=1000$. green curves: prefactor $v f_{0}=10^{8}$; factor $g_{0}=8000$ (sames sets of parameters as for Figure 7).

Increasing $\lambda$, that is increasing the overstretching, decreases the nucleation barrier and thus accelerates the kinetics. Then it may be possible to shift the time scale depending on $\lambda$ in such a way that the kinetics remains roughly the same. Such an heuristic master curve was recently established in. ${ }^{37}$ This point will be discusses further in Section 4.2.

\section{Comparison to experimental results}

Numerous experimental studies of SIC in NR have been published. Here we shall select some specific results which directly illustrate the above considerations. Several types of experiments can be discussed. The first type is related to the evolution of the melting temperature as a function of the stretching ratio or the applied stress, according to Equations (12) or (14). These data involve the chain length $N$, the maximum (crystalline) extension ratio $\lambda_{c}$ and the melting enthalpy $Q$ as material parameters. The second type is related to the lever rule, Equation (8), which contains essentially one single adjustable parameter $\lambda_{c}$. 
Kinetic evolutions may finally be discussed and, besides $\lambda_{c}$, involve also crystal-amorphous surface energies $\gamma_{s}$ and $\gamma_{e}$ and the modulus $G$ (or more generally the parameters of the hyperelastic constitutive equation in the amorphous phase).

The evolution of the melting temperature as a function of the stretching ratio or the applied stress has been measured by several authors. ${ }^{6,7}$ The data from Miyamoto et $\mathrm{al}^{7}$ are reproduced in Figure 9, together with the fitting curve obtained from Equation (12) combined with the constitutive equation $\sigma=\left(R T / v_{m} N\right)\left(\lambda-\lambda^{-2}\right)$, using the values $N=70$, $Q=4.7 \mathrm{~kJ} / \mathrm{mol}, \lambda_{c}=14.8$ and $v_{m}=6.7 \times 10^{-5} \mathrm{~m}^{3} / \mathrm{mol}$ (which corresponds roughly to one monomer). This fitting curve is similar to that shown by Miyamoto et al. ${ }^{7}$ Trabelsi et al. ${ }^{6}$ show such curves as well with similar values of the fitting parameters.

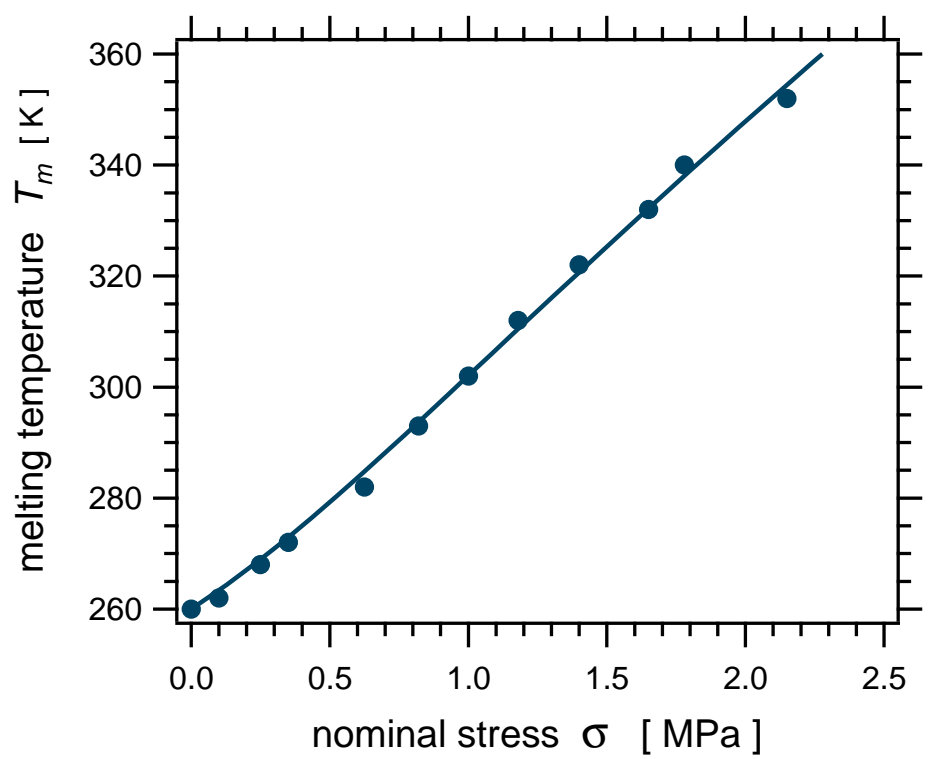

Figure 9: The melting temperature as a function of the applied nominal stress. Bullets are data reproduced from Miyamoto et al, ${ }^{7}$ the curve is a fit with equation (12) combined with $\sigma=\left(R T / v_{m} N\right)\left(\lambda-\lambda^{-2}\right)$. The following parameter values were used: $N=70, Q=4.7$ $\mathrm{kJ} / \mathrm{mol}, \lambda_{c}=14.8$ and $v_{m}=6.7 \times 10^{-5} \mathrm{~m}^{3} / \mathrm{mol}$.

The melting temperature extrapolated at zero stress $T_{m}^{*}$ reported in Figure 9 as well as by other authors is about $260 \mathrm{~K} .^{6,7}$ This temperature should be clearly distinguished from the equilibrium melting temperature of NR crystallized in the quiescent (relaxed) state and extrapolated to infinite lamellar thickness, which is commonly denoted $T_{m}^{0}$. Indeed, crystal 
morphologies are likely to be very different in either case. ${ }^{54}$ In the quiescent state, NR forms folded crystals. $T_{m}^{0}$ is determined by extrapolation using the Gibbs-Thomson equation, which contains the fold-surface energy as a parameter. ${ }^{55}$ The $T_{m}^{0}$ value determined in this way is $36^{\circ} \mathrm{C} .{ }^{55} \mathrm{~A}$ more recent estimate is about $60^{\circ} \mathrm{C} .{ }^{56}$ This quite large difference between $T_{m}^{*}$ and $T_{m}^{0}$ is likely to come from the fact that strain-induced crystals in crosslinked materials remain of nanometric sizes and that the fold energy does not contribute in a significant way. Indeed, the surface energies used here, of order $5 \times 10^{-3} \mathrm{~J}_{\mathrm{m}} \mathrm{m}^{-2}$, are significantly lower than the fold-energy value $46 \times 10^{-3}{\mathrm{~J} . \mathrm{m}^{-2}}$ proposed in ref. ${ }^{56}$

Data involving the lever rule and the kinetics are discussed in the next sections.

\subsection{The lever rule}

When considering the evolution of the stress as a function of the crystallinity, the presence of crystallites induces a dramatic self-reinforcement effect in the material. This has been extensively documented and tentatively modelled by numerous authors. ${ }^{18,23,57,58}$ During a stretching cycle, the stress first decreases at the onset of SIC relative to the stress which would be measured in a non crystallizing sample, in qualitative agreement with Equation (10). However, this decrease is quite limited, as the stress rises abruptly quite soon after SIC onset, due to the strong self-reinforcing effect of crystallites acting as solid fillers in the material.

The approach proposed here circumvent this problem by considering the relationship between the crystallinity index and the local strain in the amorphous phase $\lambda_{a}$, instead of the stress. $\lambda_{a}$ was used here as the order parameter of the SIC phase transformation, characterized by the fact that it takes two distinct values in the two coexisting crystal and amorphous phases. This relationship is described by the lever rule, Equation (8), which plays a cen-

tral role in the theory. Equation (12) or equivalently (14), which predicts the relationship between the equilibrium melting temperature and the draw ratio, is a direct consequence of this lever rule, as well as Equation (10), which predicts the variation of the stress as a 
function of the crystallinity. Equation (10), however, disregards the self-reinforcement effect mentionned above. These variations have been semi-quantitatively verified by a number of authors.

Another direct observation of the lever rule was given by Miyamoto et al. who performed stress-controlled experiments over a range of temperatures. ${ }^{7}$ They indeed obtained curves qualitatively similar to the iso-stress curve shown in green in Figure 5. However, one central message of this work is that the order parameter $\lambda_{a}$ can be directly measured in real time together with the crystallinity index by in-situ X-ray diffraction experiments. ${ }^{36,37,59}$ Such measurements thus provide direct access to the thermodynamic parameter suitable to describe the transition. Note that $\lambda_{a}$ may also be measured by NMR, though in a somehow more indirect way. ${ }^{60}$

In Section 3.3 it was assumed that the lever rule remains valid during time evolution. This statement was illustrated in two ways in reference. ${ }^{37}$ First, during a cycle performed at a given drawing rate, the draw ratio $\lambda_{a}$ can be used to predict the crystallinity $\chi$ by using Equation (8), with only one adjustable parameter, namely the extension ratio of the crystalline phase $\lambda_{c}$. This prediction can then be compared to the measured $\chi$. Good agreement was found nearly along the whole cycles, except perhaps during part of the retraction branch of the cycles, due to some creep contribution not related to crystallization.

Secondly, this statement is also evidenced by relaxation experiments such as shown in our recently published work. ${ }^{37}$ In such experiments an amorphous sample is quasi instantaneously stretched to a draw ratio $\lambda$ beyond the onset of SIC. The sample is then maintained at this fixed $\lambda$ while both the crystallinity $\chi(t)$ and amorphous draw ratio $\lambda_{a}(t)$ are recorded simultaneously as a function of time. This is illustrated in Figure 10, in which relaxation data obtained at different values of $\lambda$ are shown. The quantity $1-\chi(t)$ (the amorphous fraction) is plotted as a function of the quantity $\left(\lambda_{c}-\lambda\right) /\left(\lambda_{c}-\lambda_{a}(t)\right)$. The line illustrates the predicted one-to-one relationship between both quantities. 


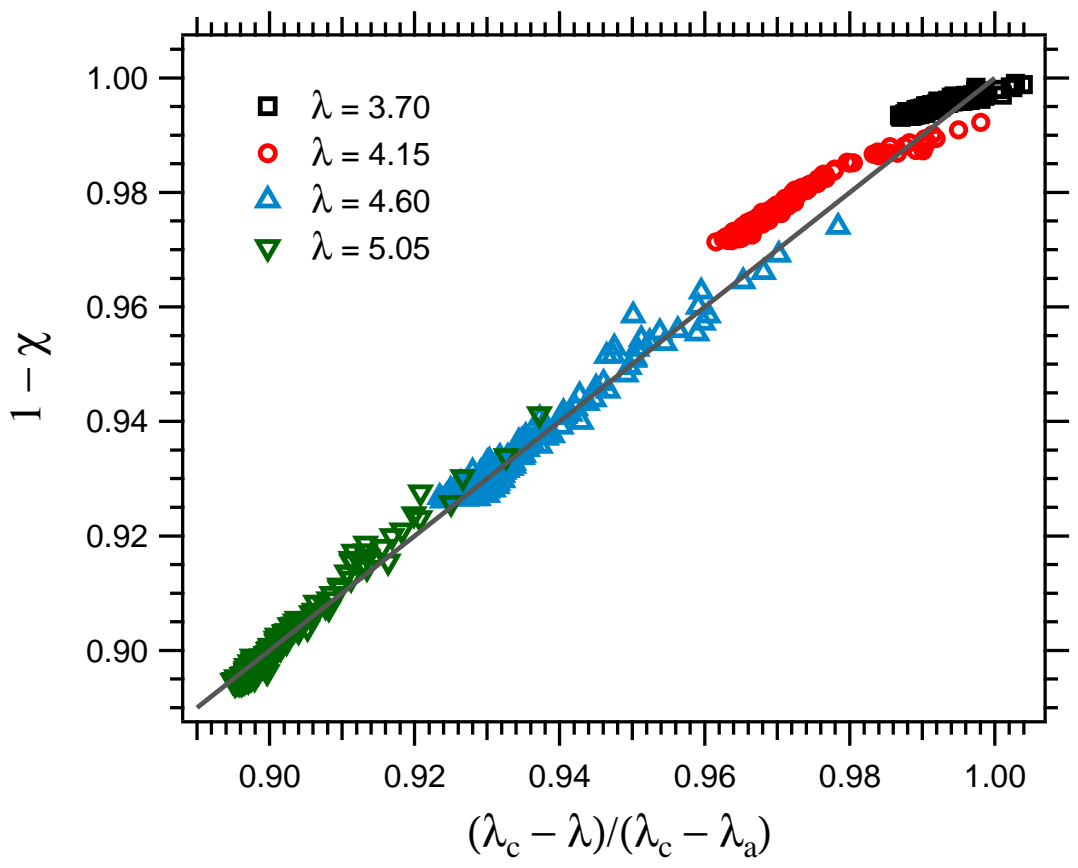

Figure 10: The amorphous fraction $1-\chi(t)$ as a function of the quantity $\left(\lambda_{c}-\lambda\right) /\left(\lambda_{c}-\lambda_{a}(t)\right)$ during relaxation experiements done at various $\lambda$ values as indicated. The line illustrates the predicted one-to-one relationship between both quantities according to the lever rule equation (8). The time scale goes from top right to left along these curves. Data are the same as in ref. ${ }^{37}$ 


\subsection{Crystallization kinetics}

Two situations may be discussed. First, as reported by several authors, the SIC onset depends on the draw rate during a stretching cycle. ${ }^{5,26,37,61,62}$ Conversely, the stretching ratio at melting $\lambda_{\text {melt }}$ does not seem to depend signifciantly on the rate. ${ }^{37}$ Qualitatively both observations are in full agreement with the general framework presented above.

Assuming, as noted above, that the lever rule Equation (8) remains valid during timedependent situations, the following equation is derived:

$$
\frac{d \lambda_{a}}{d t}=\frac{d \lambda}{d t}-\left(\lambda_{c}-\lambda_{a}\right) \frac{d \chi}{d t}
$$

During a stretching cycle in which $d \lambda / d t$ is constant, after a short transient regime, the crystallization rate is fixed by the drawing velocity in such a way that $\lambda_{a}$ remains constant. This effect has been denoted as strain regulation effect. ${ }^{37}$ Thus $\lambda_{\text {onset }}$ is such that the initial rate (rate at onset) $\omega=d \chi / d t$, which itself depends on $\lambda_{\text {onset }}$, obeys the equation

$$
\frac{d \lambda}{d t}=\left(\lambda_{c}-\lambda_{\text {onset }}\right) \omega
$$

From equations (24) and (25) the rate $\omega$ is expressed as a function of $\lambda_{\text {onset }}$ as:

$$
\omega \sim \exp \left[-\frac{8 \pi \gamma_{s}^{2} \gamma_{e}}{k_{B} T G^{2}\left(\lambda_{c}-\lambda_{\text {onset }}\right)^{2}\left(\lambda_{\text {onset }}-\lambda_{\text {melt }}\right)^{2}}\right]
$$

Values of the $\lambda_{\text {onset }}$ values measured during drawing at various strain rates are reported in Figure 11. The data were fitted using combined equations 32 and 33. The values $\lambda_{c}=14.8$ and $\lambda_{\text {melt }}=2.63$ were fixed. The obtained adjusted value for the factor $g_{0}=8 \pi \gamma_{s}^{2} \gamma_{e} /\left(k_{B} T G^{2}\right)$ is 3000 , and this value is within the anticipated range, see Section 3.3. The corresponding surface energies are of order $5 \times 10^{-3} \mathrm{~N} \cdot \mathrm{m}^{-1}$.

The second type of experiment that we shall consider is the follow-up of the kinetics under a fixed $\lambda$ value, as already discussed in Section 4.1. While $\lambda$ is maintained fixed, 


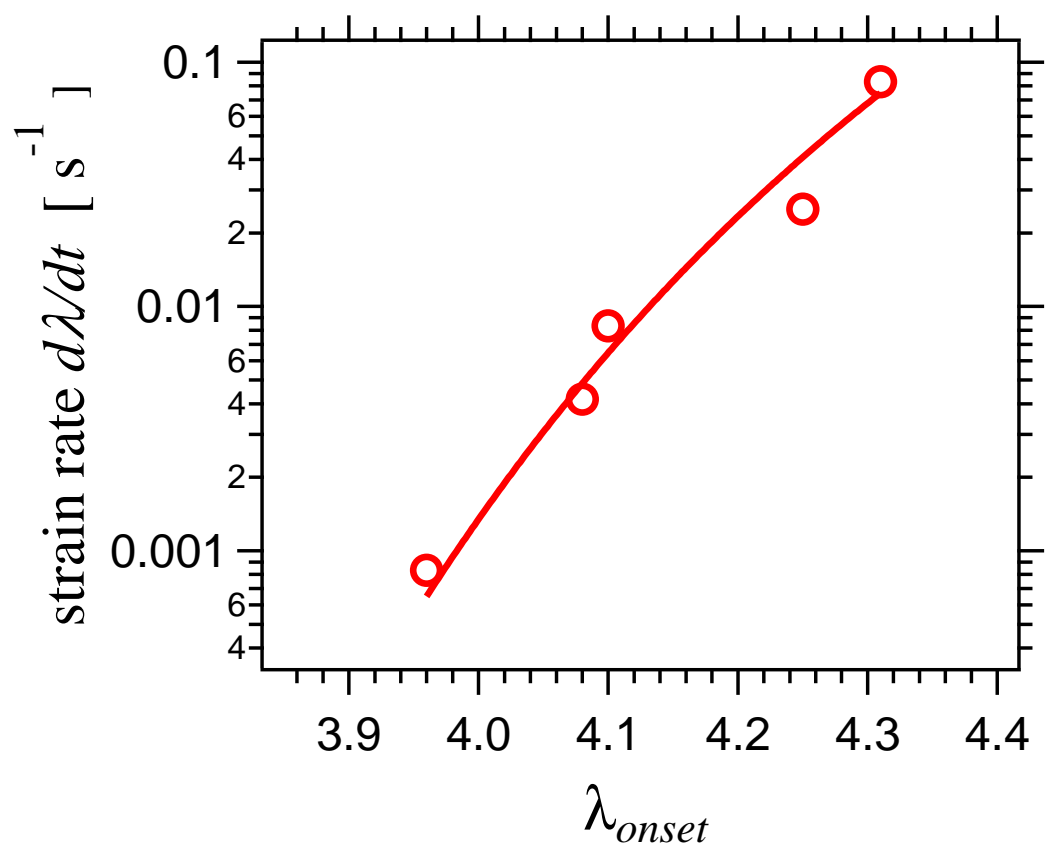

Figure 11: The draw ratio at SIC onset $\lambda_{\text {onset }}$ (in abscissa) measured when stretching at a given strain rate (in ordinate). The data are the same as in ref. ${ }^{37}$ The curve is a fit using equations (32) and (33). The values $\lambda_{c}=14.8$ and $\lambda_{\text {melt }}=2.63$ were fixed and the value $g_{0}=3000$ was obtained.

both the crystallinity $\chi(t)$ and amorphous draw ratio $\lambda_{a}(t)$ are recorded simultaneously as a function of time.

This is illustrated in Figure 12, in which the quantity $\chi(t) / \chi_{e q}$ (in which $\chi_{e q}$ is calculated for each value of $\lambda$ by using the lever rule $\left.\chi_{e q}=\left(\lambda-\lambda_{\text {melt }}\right) /\left(\lambda_{c}-\lambda_{\text {melt }}\right)\right)$ is shown as a function of time for different values of $\lambda$. The full curves are obtained by solving equation (30) using for the factor $g_{0}=8 \pi \gamma_{s}^{2} \gamma_{e} /\left(k_{B} T G^{2}\right)$ the same value 3000 as found previously, without further adjustment. A good qualitative agreement is obtained. From a quantitative point of view, the adjustment is not much better than the one proposed e.g. in ref. ${ }^{27}$ One major point to emphasize here is the correct order of magnitude of the factors involved in the model. Basically, besides the prefactor $f_{0}$ which scales the overall nucleation rate and is related to chain dynamics, the model contains only one unknown adjustable parameter, namely the combination $\gamma_{s}^{2} \gamma_{e}$ in the factor $g_{0}$ in the nucleation barrier, related to the crystal - amorphous interfacial energies. Reported values for the interfacial energies for e.g. polyolefins are of 
order $2-2.5 \times 10^{-2} \mathrm{~N} / \mathrm{m} .{ }^{41}$ However, the values relevant to $\mathrm{SIC}$ cannot be directly inferred from quiescent thermal crystallization, as crystal morphologies may be completely different in

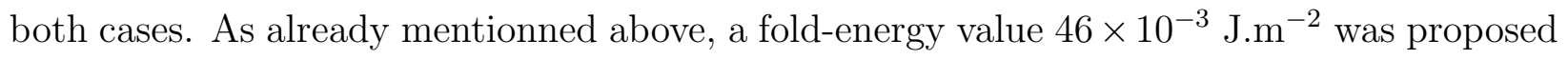
in ref. ${ }^{56}$ As noted above, from the value $g_{0}=3000$, values of order $5 \times 10^{-3}$ are obtained for surface energies. This value is noticeably smaller than the values mentionned above, but is realistic.

To take into account the disorder in the system, a distribution of local strain values should perhaps be considered. This is equivalent to considering a distribution of $\lambda_{\text {melt }}$ values. Indeed, introducing a log-normal distribution of $\lambda_{\text {melt }}$ centered on the measured, previosuly considered value $\lambda_{\text {melt }}=2.63$, significantly improves the adjustement to experimental data, as shown by the dashed curves in Figure 12.

It results from the general evolution of the curves shown in Figure 12 that an apparent, approximate master curve may be drawn by renormalizing the time scale with a $\lambda$-dependent factor. This is in agreement with the basic outcome of the nucleation model that the crystallization rate depends on $\lambda$ (or more precisely on the overstretching parameter $\lambda-\lambda_{\text {melt }}$ ), as it was already mentionned in Section 3.3. Such a master curve was recently proposed in. ${ }^{37}$

The data of Figure 12 are plotted in Figure 13 as a function of a reduced time scale $a(\lambda) t$ in which the shift factor $a(\lambda)$ is determined to optimize the overlap of the different partial curves. The curve measured at $\lambda=4.60$ was chosen as reference. Experimental data overlap nearly perfectly, except perhaps for the curve at $\lambda=5.05$. Curves calculated with a distribution $\lambda_{\text {melt }}$ (dashed curves in Figure 12) are also plotted in Figure 13 using the same set of shift factors. As explained above, they do not overlap but show the general tendency that time scales related to different values of $\lambda$ roughly correspond to each other.

The values of the shift factor $a(\lambda)$ used to draw figure 13 are reported in Table 1 and in Figure 14. The strain rate vs $\lambda_{\text {onset }}$ data in Figure 11 are also shown again in Figure 14. The comparision of both sets of data shows that the general relationship between the draw ratio and the time scale is indeed similar in both types of experiments. Note that the perfect 


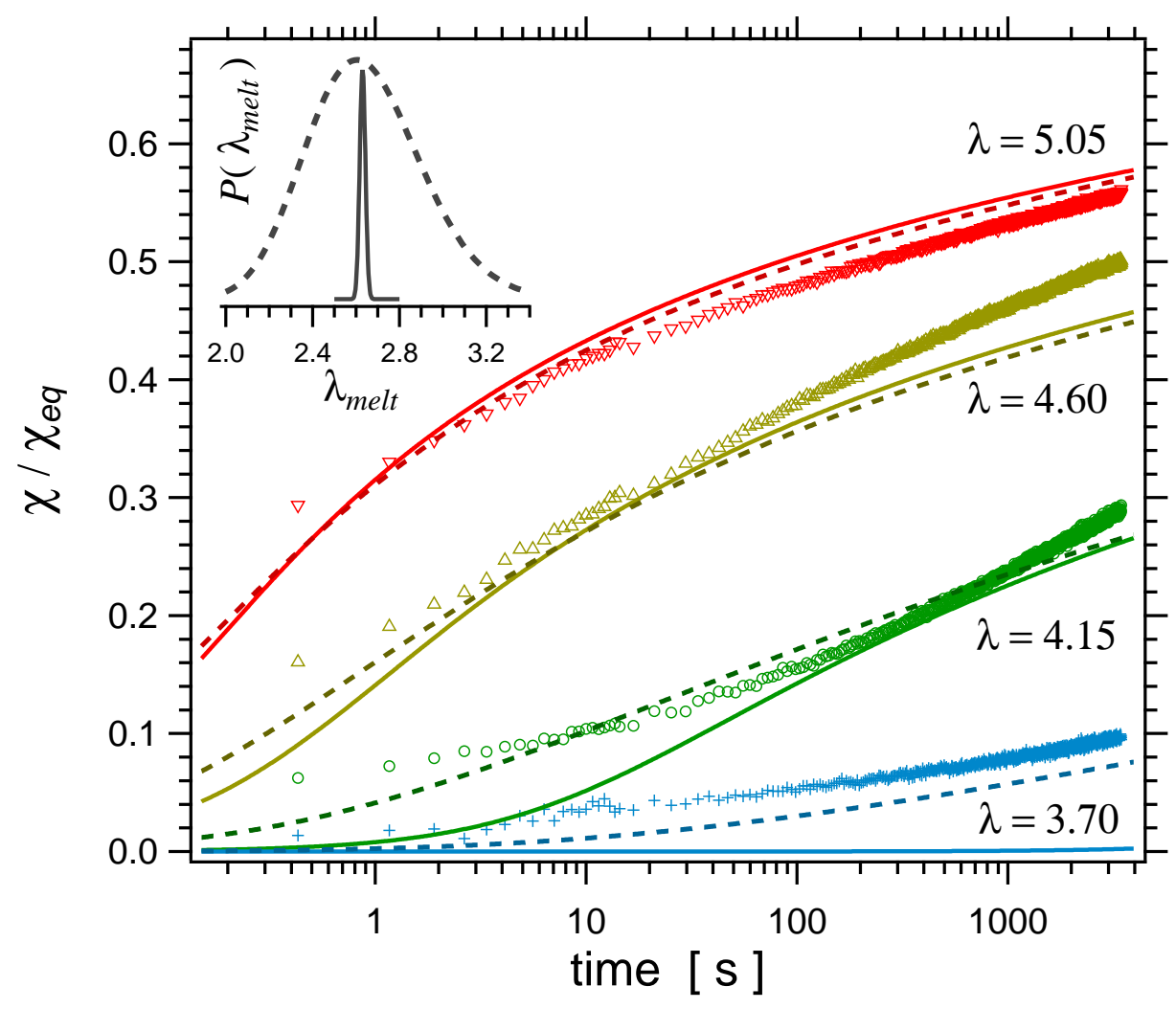

Figure 12: The quantity $\chi(t) / \chi_{e q}$ as a function of time for various $\lambda$ values as indicated. Same data as in ref. ${ }^{37}$ Curves correspond to equation (30) using $g_{0}=8 \pi \gamma_{s}{ }^{2} \gamma_{e} /\left(k_{B} T G^{2}\right)=3000$ as previously. Other parameters are $f_{0}=10^{2}, \lambda_{c}=14.8$. A log-normal distribution was used for $\lambda_{\text {melt }}$, centered around $\lambda_{\text {melt }}=2.63$. Full curves: distribution width 0.005 (very narrow distribution, full curve in inset); dashed curve: width 0.1 (wide distribution, dashed curve in inset). 


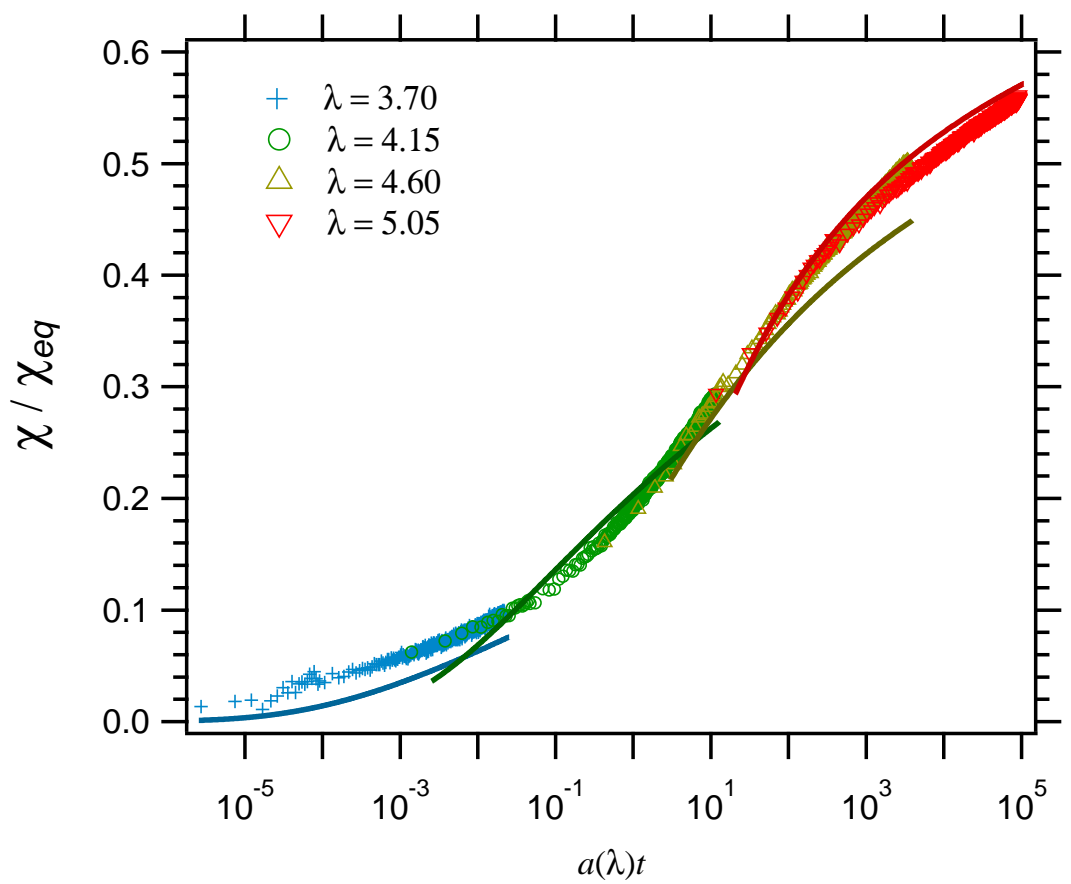

Figure 13: The quantity $\chi(t) / \chi_{e q}$ as a function of the reduced time-scale $a(\lambda) t$ for various $\lambda$ values as indicated. Same data as in $\operatorname{ref}^{37}$ and Figure 12. Curves: equation (30) with $8 \pi \gamma_{s}{ }^{2} \gamma_{e} /\left(k_{B} T G^{2}\right)=3000, v f_{0}=10^{2}, \lambda_{c}=14.8$ and log-normal distribution for $\lambda_{m e l t}$ with $\left\langle\lambda_{\text {melt }}\right\rangle=2.63$ and width $w=0.1$ (distribution shown by dashed curve in inset of Figure 12). 
coincidence of both sets of data on the vertical scale is fortuitous.

An approximate way of analysing the values of the shift factor $a(\lambda)$ is to compute the initial nucleation rates corresponding to the imposed $\lambda$ values. This was done using Equation (25). The curve in Figure 14 was obtained with the value $g_{0}=5200$ and a distribution for $\lambda_{\text {melt }}$ of width $w=0.06$, still using the same values $\lambda_{c}=14.8$ and $\lambda_{\text {melt }}=2.63$.

As the crystallization rate is not constant along the time evolution, this is only a rough approximation of the whole behavior. Parameters used to obtain the curve in Figure 14 are slightly different from those used previously. However, this analysis once again shows that the general relationship between the draw ratio and the time scale can be understood on the basis of the proposed model.

Table 1: The shift factor $a(\lambda)$ used to construct the master curves in ref. ${ }^{37} \lambda_{\text {melt }}=2.63$. $\Delta \lambda$ is the initially imposed over-stretching value.

\begin{tabular}{ccc}
\hline$\lambda$ & $\Delta \lambda$ & $a(\lambda)$ \\
\hline 3.70 & 1.07 & $6 \times 10^{-6}$ \\
4.15 & 1.52 & $3.26 \times 10^{-3}$ \\
4.60 & 1.97 & 1 \\
5.05 & 2.42 & 27.16 \\
\hline
\end{tabular}

\section{Discussion}

The theory presented in Section 2.2 captures the essential features and physical mechanisms of SIC in a simple way. One key feature in the model is the lever rule as expressed in Equation (8). Special emphasis was put on the importance of considering and elaborating strategies to directly measuring the local draw ratio of the amorphous phase $\lambda_{a}$, which was introduced as the order parameter of SIC. Indeed, it has been shown experiementally that $\lambda_{a}$ indeed exhibits a plateau during SIC. Conversely the measured stress under SIC does not show a plateau. After a relatively tiny downward inflexion, it exhibits strong selfreinforcement. This is due to the reinforcement effect brought by crystallites acting as rigid reinforcing fillers. Describing this reinforcing effect in a quantitative way is a matter of 


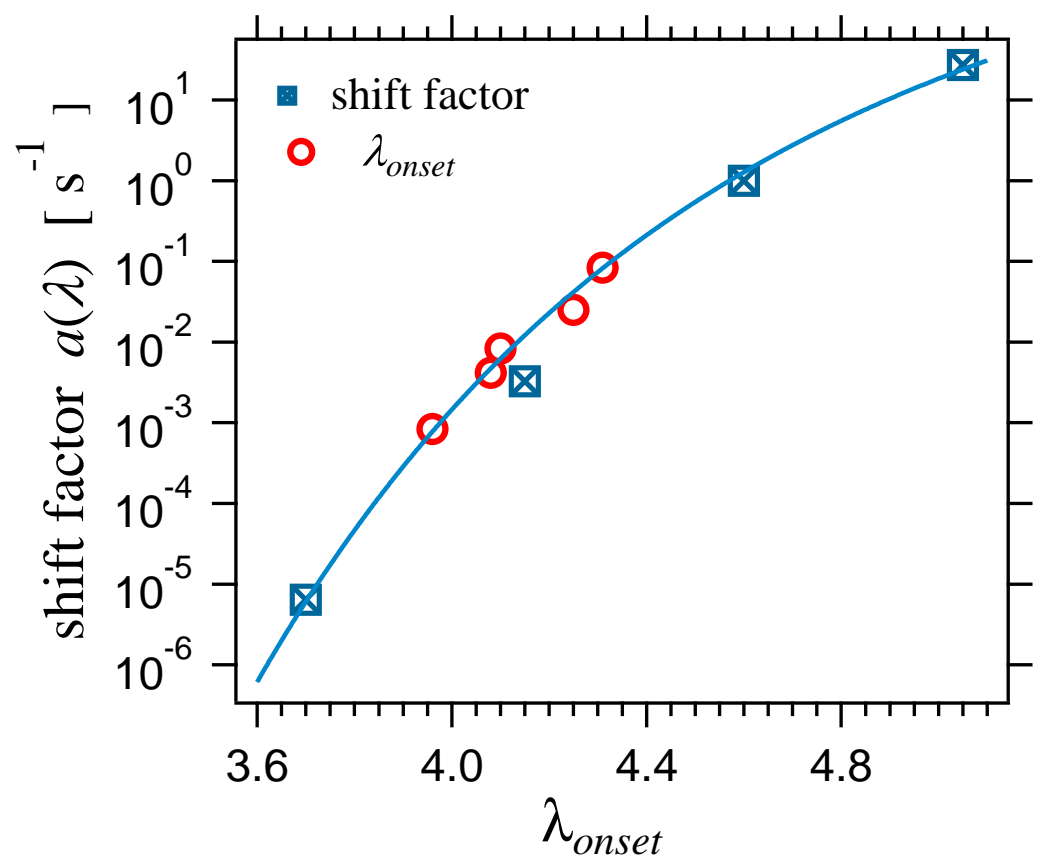

Figure 14: Blue squares: the shift factor $a(\lambda)$ used to construct the master curves in ref. ${ }^{37}$ Symbols are from experimental data, the curve was calculated from Equation (25) with parameters $\lambda_{\text {melt }}=2.63, \lambda_{c}=14.8, g_{0}=5200$ and width of the $\lambda_{\text {melt }}$ distribution $w=0.06$ . Data for $\lambda_{\text {onset }}$ vs strain rate (same data as in Figure 11) are also reported for comparison (red circles). 
involved modelling, which was not addressed in the present work. ${ }^{58}$

As regards the analysis of kinetic phenomena, it was assumed that SIC is entirely driven by nucleation, and that the contribution of crystal growth is negligible. It follows that one key parameter is the height of the nucleation barrier, as expressed e.g. in Equation (24). This nucleation barrier contains the modulus of the amorphous phase, which is can be measured directly, and contains a factor related to the crystal-amorphous interfacial energies. This is the only unknown factor in the expression of the nucleation barrier. One main difference with commonly considered thermal crystallization is that the crystallization rate evolves along the crystallization process due to relaxation of the driving force. Recently obtained data on the crystallization kinetics were compared to the predictions of the model. It was first demonstrated that the general trend of the crystallization kinetics can be reproduced in a satisfactory way, with perfectly realistic values of the nucleation barrier. Nevertheless, the fact that perfect superposition is observed experimentally when drawing a master curve from the data in Figure 12 (see Figure 13), while this is not what is predicted, is intriguing.

The model is however clearly oversimplified in several aspects and some important questions remain elusive. First, the model is one-dimensional. As a first approach, this is justified by the experimental observation that crystals generated during SIC are strongly oriented, with chains along the tensile direction, as reported in numerous references.

A more basic question is the following one. In contrast to a simple substance, crystallization is never complete in a polymer, and this of course remains true for SIC. Then, which phase equilibrium should actually be considered? Should equilibrium between the amorphous and crystalline phases be considered, as was implicitly assumed in this work, or between a fully amorphous and a semi-crystalline phase be assumed, as suggested by the observation of an inverse striction phenomenon on retraction. ${ }^{63}$

It results from the model that the critical nucleation sizes (Equation (19)) shall evolve during relaxation experiments. The critical nucleation size $\xi_{c}$ is estimated to be $\xi_{c} \approx 4$ $\mathrm{nm}$ at the beginning of relaxation, assuming $\Delta \sigma \approx 1 \mathrm{MPa}$. It then increases progressively 
as relaxation proceeds, as noted above. This is comparable, though a little bit smaller, than crystal dimensions measured in comparable conditions, which amounts to 5 to $20 \mathrm{~nm}$, depending on the considered direction. Actually this comparison is a strong argument to state that crystallization is mostly driven by nucleation. Note also that the heterogeneity in the crystallite population has been proposed to be an important factor to explain the peculiar features of SIC. ${ }^{53,64}$ Besides, in crosslinked Natural Rubber (NR) elastomers as commonly studied in the literature, the assumption that SIC is entirely driven by nucleation and that the contribution of crystal growth is negligible may be further justified as the presence of crosslinks must inhibit crystal growth. The dependence of the overall crystallization kinetics on the crosslink density shall be investigated in a forthcoming work.

Discrepancies between the model and experimental observations may come from the contribution of heterogeneous nucleation, as crosslinked NR usually contain additives which may act as nucleating agents, such as e.g. sulfur micrometric aggregates, ZnO particles and stearic acid crystals. Heterogeneous nucleating agents may have an impact at small overstretching values. Indeed, crystallization kinetics seems to be faster than predicted at low overstretching, i.e. low $\lambda$ values (see Figure 12). A distribution of interfacial energies $\gamma$ should perhaps be considered to take this into account. The approach proposed here may then allow elucidating the effect of filler particles in reinforced NR materials. In the presence of carbon black (CB), a downward shift of the draw ration at SIC onset is generally observed. ${ }^{60,65}$ This has been interpreted as mostly due to a nucleating effect of fillers ${ }^{60}$ or alternatively to local strain amplification. ${ }^{65}$ The relative contributions of either effect in various circumstances or measuring conditions remain to be fully clarified. A distribution of $\lambda_{\text {melt }}$ was introduced, based on the idea that the local disorder in the material in terms of local strain should be considered. Using a single log-normal distribution should be considered as a preliminary attempt, which illustrates the relevance of the model. Using Tikhonov regularization techniques might be used to access experimentally determined distributions of local strains. 


\section{Conclusion}

We have given a unified picture of Strain Induced Crystallization (SIC) in Natural Rubber in which the basic physical ingredient of the seminal theory by Flory, that is, the entropic relaxation of amorphous chains concomitant to SIC, is implemented in the form of a simple phase equilibrium, similar to e.g. liquid-gas equilibrium of a pure substance. The order parameter describing this phase transformation is the local draw ratio. This emphasizes the

key importance of directly measuring this quantity, which was proposed recently. ${ }^{36,37,59}$ At a given temperature (above the melting temperature in the relaxed state), there is a critical value $\lambda_{\text {melt }}$ at which crystalline and amorphous phases coexist at equilibrium. It follows from the model that the driving force for strain induced crystallization crystallization is the overstretching parameter $\Delta \lambda=\lambda-\lambda_{\text {melt }}$.

The evolution of the crystallinity index as a function of the strain (equivalently, of the over-stretching) when stretching a sample at a constant drawing rate is conceptually analogous to the evolution of the crystallinity index as a function of temperature (equivalently, of the undercooling) when cooling a sample at a fixed cooling rate. However, the evolution of the crystallintiy index in a sample maintained at a fixed macroscopic over-stretching parameter is not exactly analogous to isothermal crystallization, because the draw ratio (or over-stretching) of the amorphous phase, which determines the driving force, relaxes as crystallinity increases, whereas in isothermal crystallization, temperature is constant.

A kinetic equation for the crystallization rate was proposed based on standard homogeneous nucleation theory. The dependence of the thermodynamic barrier for nucleation on the overstretching parameter $\Delta \lambda$ was inferred. The results predicted from the model compare well to experiemental data with realistic values of the nucleation barrier. 


\section{References}

(1) Katz, J. R. Röntgenspektrographische Untersuchungen am gedehnten Kautschuk und ihre mögliche Bedeutung fr das Problem der Dehnungseigenschaften dieser Substanz. Die Naturwissenschaften 1925, 19, 410-416.

(2) Flory, P. J. Thermodynamics of Crystallization in High Polymers. I Crystallization Induced by Stretching. J. Chem. Phys. 1947, 15, 397-408.

(3) Mitchell, J. C.; DJ Meir, D. J. Rapid Stress-Induced Crystallization in Natural Rubber. J. Polym. Sci. Pol. Chem. 1968, 6, 1689-1703.

(4) Luch, D.; Yeh, G. S. Y. Strain-induced crystallization of natural rubber. Bulletin of the American Physical Society 1971, 16, 366-\&.

(5) Toki, S.; Fujimaki, T.; Okuyama, M. Strain-induced crystallization of natural rubber as detected real-time by wide-angle X-ray diffraction technique. Polymer 2000, 41, $5423-5429$.

(6) Trabelsi, S.; Albouy, P.-A.; Rault, J. Crystallization and Melting Processes in Vulcanized Stretched Natural Rubber. Macromolecules 2003, 36, 7624-7639.

(7) Miyamoto, Y.; H Yamao, H.; Sekimoto, K. Crystallization and Melting of Polyisoprene Rubber under Uniaxial Deformation. Macromolecules 2003, 36, 6462-6471.

(8) Tosaka, M.; Kohjiya, S.; Murakami, S.; Poompradub, S.; Ikeda, Y.; Toki, S.; Sics, I.; Hsiao, B. S. Effect of network-chain length on strain-induced crystallization of NR and IR vulcanizates. Rubber Chem. Technol. 2004, 77, 711-723.

(9) Tosaka, M. Strain-induced crystallization of crosslinked natural rubber as revealed by X-ray diffraction using synchrotron radiation. Polym. J. 2007, 39, 1207. 
(10) Poompradub, S.; Tosaka, M.; Kojiya, S.; Ikeda, Y.; Toki, S.; Sics, I.; Hsiao, B. S. Mechanism of strain-induced crystallization in filled and unfilled natural rubber vulcanizates. J. Appl. Phys. 2005, 9\%, 103529.

(11) Chenal, J.-M.; Chazeau, L.; Guy, L.; Bomal, Y.; Gauthier, C. Molecular weight between physical entanglements in natural rubber: A critical parameter during strain-induced crystallization. Polymer 2007, 48, 1042-1046.

(12) Chenal, J.-M.; Chazeau, L.; Bomal, Y.; Gauthier, C. New insights into the cold crystallization of filled natural rubber. J. Polym. Sci., Part B: Polym. Phys. 2007, 45, $955-962$.

(13) Huneau, B. Strain-Induced Crystallization of Natural Rubber: A Review of X-Ray Diffraction Investigations. Rubber Chem. Technol. 2011, 84, 425-452.

(14) Demassieux, Q.; Berghezan, D.; Cantournet, S.; Prudhon, H.; Creton, S. Temperature and Aging Dependence of Strain-Induced Crystallization and Cavitation in Highly Crosslinked and Filled Natural Rubber. Journal of Polymer Science, Part B: Polymer Physics 2019, 57, 780-793.

(15) Yamamoto, M.; White, J. L. Theory of Deformation and Strain-Induced Crystallization of an Elastomeric Network Polymer. J. Polym. Sci. Pol. Chem. 1971, 9, 1399-1415.

(16) Gaylord, R. J. A Theory of Stress-Induced Crystallization of Crosslinked Polymeric Networks. J. Polym. Sci. Pol. Phys. 1976, 14, 1827-1837.

(17) Smith, K. J. Crystallization of networks under stress. Polymer Engineering and Science 1976, 16, 168-175.

(18) Wu, W. L. A Thermodynamic Approach to the Stress-Induced Crystallization in Crosslinked Rubbers. J. Polym. Sci. Pol. Phys. 1978, 16, 1671-1683. 
(19) Dargazany, R.; Khiêm, V. N.; Poshtan, E. A.; Itskov, M. Constitutive modeling of strain-induced crystallization in filled rubbers. Phys. Rev. E 2014, 89, 022604.

(20) Klüppel, M.; Plagge, J. A Theory Relating Crystal Size, Mechanical Response, and Degree of Crystallization in Strained Natural Rubber. Macromolecules 2018, 51, 37113721.

(21) Kroon, M. A constitutive model for strain-crystallizing rubberlike materials. Mech. Mater. 2010, 42, 873-885.

(22) Mistry, S. J.; Govindjee, S. A micro-mechanically based continuum model for straininduced crystallization in natural rubber. Int. J. Solids Struct. 2014, 51, 530-539.

(23) Guilié, J.; Thien-Nga, L.; Le Tallec, P. Micro-sphere model for strain-induced crystallisation and three-dimensional applications. Journal of the Mechanics and Physics of Solids 2015, 81, 58-74.

(24) Laghmach, R.; Candau, N.; Chazeau, L.; Munch, E.; Biben, T. Phase field modelling of strain induced crystal growth in an elastic matrix. J. Chem. Phys. 2014, 142, 244905.

(25) Gros, A.; Huneau, B.; Verron, E.; Tosaka, M. A physically-based model for straininduced crystallization in natural rubber. Part I: Life cycle of a crystallite. Journal of the Mechanics and Physics of Solids 2019, 125, 164-177.

(26) Brüning, K.; Schneider, K.; Roth, S. V.; Heinrich, G. Kinetics of Strain-Induced Crystallization in Natural Rubber Studied by WAXD: Dynamic and Impact Tensile Experiments. Macromolecules 2012, 45, 7914-7919.

(27) Brüning, K.; Schneider, K.; Roth, S. V.; Heinrich, G. Kinetics of strain-induced crystallization in natural rubber: A diffusion-controlled rate law. Polymer 2015, 72, 52-58. 
(28) Pannier, Y.; Proudhon, H.; Mocuta, C.; Thiaudiere, D.; Cantournet, S. In situ multiaxial loading frame to probe elastomers using X-ray scattering. Journal of Synchrotron Radiation 2011, 18, 907-911.

(29) Caimmi, F.; Calabro, R.; Briatico-Vangosa, F.; Marano, C.; Rink, M. Toughness of natural rubber compounds under biaxial loading. Engineering Fracture Mechanics 2015, $149,250-261$.

(30) Chen, X.; Meng, L.; Zhang, W.; Ye, K.; Xie, C.; Wang, D.; Wei Chen, W.; Nan, M.; Wang, S.; Li, L. Frustrating Strain-Induced Crystallization of Natural Rubber with Biaxial Stretch. ACS Appl. Mater. Interfaces 2019, 11, 47535-47544.

(31) Landau, L. D.; Lifchitz, E. M. Statistical Physics; MIR Editions: Masocw, 1967.

(32) Plagge, J.; Klüppel, M. A Theory Relating Crystal Size, Mechanical Response, and Degree of Crystallization in Strained Natural Rubber. Macromolecules 2018, 51, 37113721.

(33) Treloar, L. R. G. The Physics of Rubber Elasticity; Clarendon Press, Oxford, England, 1975.

(34) Erman, B.; Mark, J. E. Structures and Properties of Rubberlike Networks; Oxford University Press New York, 1997.

(35) De Rosa, C.; Auriemma, F. Crystals and Crystallinity in Polymers; Wiley: Hoboken, New Jersey, 2014.

(36) Albouy, P.-A.; Sotta, P. In Polymer Crystallization. From Chain Microstructure to Processing; Auriemma, F., Alfonso, G. C., De Rosa, C., Eds.; Adv. Polym. Sci.; Springer International Publishing, 2017; Vol. 277; pp 167-205.

(37) Albouy, P.-A.; Sotta, P. Draw ratio at onset of strain-induced crystallization in crosslinked natural rubber. Macromolecules 2020, 53, 992-1000. 
(38) Le Cam, J.-B. Energy storage due to strain-induced crystallization in natural rubber: The physical origin of the mechanical hysteresis. Polymer 2017, 127, 166-173.

(39) Le Cam, J.-B. Strain-induced crystallization in rubber: A new measurement technique. Strain 2018, 54, e12256.

(40) Cluzel, P.; Lebrun, A.; Heller, C.; Lavery, R.; Viovy, J. L.; Chatenay, D.; Caron, F. DNA: An extensible molecule. Science 1996, 271, 792-794.

(41) Mandelkern, L. Crystallization of Polymers: Second Edition. Volume 2, Kinetics and Mechanisms; Cambridge University Press, 2004.

(42) Strobl, G. R. The physics of Polymers. Concepts for Understanding Their Strucutres and Behavior; Springer-verlag: Berlin Heidelberg, 2007.

(43) Sajkiewicz, P. Transient and Athermal Effects in the Crystallization of Polymers. I. Isothermal Crystallization. Journal of Polymer Science: Part B: Polymer Physics 2002, 40, 1835-1849.

(44) Sajkiewicz, P. Transient and Athermal Effects in the Crystallization of Polymers. II. Nonisothermal Crystallization. Journal of Polymer Science: Part B: Polymer Physics 2003, 41, 68-79.

(45) Reiter, G.; Sommer, J.-U. Polymer Crystallization; Springer-Verlag Berlin Heidelberg, 2003.

(46) Schick, C. Differential scanning calorimetry (DSC) of semicrystalline polymers. Anal Bioanal Chem 2009, 395, 1589-1611.

(47) Cormia, R. L.; Price, F. P.; Turnbull, D. Kinetics of Crystal Nucleation in Polyethylene. Journal of Chemical Physics 1962, 37, 1333-1340. 
(48) Steinhart, M.; Goering, P.; Dernaika, H.; Prabhukaran, M.; Goesele, U.; Hempel, E.; Thurn-Albrecht, T. Coherent kinetic control over crystal orientation in macroscopic ensembles of polymer nanorods and nanotubes. Physical Review letters 2006, 97, 027801.

(49) Ziabicki, A. J. Generalized Theory of Nucleation Kinetics. 4. Nucleation as Diffusion in the Space of Cluster Dimensions, Positions, Orientations, and Internal Structure. J. CHem. Phys. 1986, 85, 3042-3057.

(50) Hoffman, J. D. Thermodynamic Driving Force in Nucleation and Growth Processes. Journal of Chemical Physics 1958, 29, 1192-1193.

(51) Chew, S.; Griffiths, J. R.; Stachurski, Z. H. The crystallization kinetics of polyethylene under isothermal and non-isothermal conditions. Polymer 1989, 30, 874-881.

(52) Tobin, M. C. Theory of Phase Transition Kinetics with Growth Site Impingement. I. Homogeneous Nucleation. Journal of Polymer Science 1974, 12, 399-406.

(53) Candau, N.; Laghmach, R.; Chazeau, L.; Chenal, J.-M.; Gauthier, C.; Biben, T.; Munch, E. Temperature dependence of strain-induced crystallization in natural rubber: On the presence of different crystallite populations. Polymer 2015, 60, 115-124.

(54) Chen, P.; Lin, Y.; Zhao, J.; Meng, L.; Wang, D.; Chen, W.; Li, L. Reconstructing the mechanical response of polybutadiene rubber based on micro-structural evolution in strain-temperature space: entropic elasticity and strain-induced crystallization as the bridges. Soft Matter 2020, 16, 447-455.

(55) Dalal, E. N.; Taylor, K. D.; Philips, P. J. The equilibrium melting temperature of cis-polyisoprene. Polymer 1983, 24, 1623-1630.

(56) Tosaka, M.; Kumagawa, D. Reexamination of the equilibrium melting temperature of natural rubber crystals. Polymer Journal 2019, 52, 255-259. 
(57) Tosaka, M.; Kojiya, S.; Ikeda, Y.; Toki, S.; Hsiao, B. S. Molecular orientation and stress relaxation during strain-induced crystallization of vulcanized natural rubber. Polym. J. 2010, 42, 474-481.

(58) Gros, A.; Verron, E.; Huneau, B. A physically-based model for strain-induced crystallization in natural rubber. Part II: Derivation of the mechanical model. Journal of the Mechanics and Physics of Solids 2019, 125, 255-275.

(59) Albouy, P.-A.; Vieyres, A.; Pérez-Aparicio, R.; Sanséau,; Sotta, P. The impact of straininduced crystallization on strain during mechanical cycling of cross-linked natural rubber. Polymer 2014, 55, 4022-4031.

(60) Rault, J.; Marchal, J.; Judeinstein, P.; Albouy, P. A. Stress-induced crystallization and reinforcement in filled natural rubbers: H-2 NMR study. Macromolecules. 2006, 39, 8356-8368.

(61) Tosaka, M.; Senoo, K.; Sato, K.; Noda, M.; Ohta, N. Detection of fast and slow crystallization processes in instantaneously-strained samples of cis-1.4-polyisoprene. Polymer 2012, 53, 864-872.

(62) Candau, N.; Laghmach, R.; Chazeau, L.; Chenal, J.-M.; Gauthier, C.; Biben, T.; Munch, E. Influence of strain rate and temperature on the onset of strain induced crystallization in natural rubber. Eur. Polym. J. 2015, 64, 244-252.

(63) Albouy, P.-A.; Marchal, J.; Rault, J. Chain orientation in natural rubber, Part I: The inverse yielding effect. Eur. Phys. J. 2006, E17, 247-259.

(64) Candau, N.; Laghmach, R.; Chazeau, L.; Chenal, J.-M.; Gauthier, C.; Biben, T.; Munch, E. Strain-Induced Crystallization of Natural Rubber and Cross-Link Densities Heterogeneities. Macromolecules 2014, 47, 5815-5824. 
(65) Dupres, S.; Long, D. R.; Albouy, P.-A.; Sotta, P. Local Deformation in Carbon BlackFilled Polyisoprene Rubbers Studied by NMR and X-ray Diffraction. Macromolecules 2009, 42, 2634-2644. 